\title{
THE CLOSURE DIAGRAM FOR NILPOTENT ORBITS OF THE REAL FORM EIX OF $\mathrm{E}_{8}{ }^{*}$
}

\author{
DRAGOMIR Ž. ĐOKOVIĆ ${ }^{\dagger}$
}

\begin{abstract}
Let $\mathcal{O}_{1}$ and $\mathcal{O}_{2}$ be adjoint nilpotent orbits in a real semisimple Lie algebra. Write $\mathcal{O}_{1} \geq \mathcal{O}_{2}$ if $\mathcal{O}_{2}$ is contained in the closure of $\mathcal{O}_{1}$. This defines a partial order on the set of such orbits, known as the closure ordering. We determine this order for the noncompact nonsplit real form of the simple complex Lie algebra $E_{8}$.
\end{abstract}

1. Introduction. The closure diagrams for adjoint nilpotent orbits in noncompact real forms of $F_{4}$ and $G_{2}$ were determined in [9], for $E_{6}$ in [10], and for the noncompact and nonsplit real forms of $E_{7}$ in [11]. In this paper we handle the noncompact and nonsplit real form of $E_{8}$.

By $\mathfrak{g}$ we denote a simple complex Lie algebra of type $E_{8}$, by $\mathfrak{g}_{0}$ the real form of $\mathfrak{g}$ of type EIX, and by $G$ (respectively $G_{0}$ ) the adjoint group of $\mathfrak{g}$ (respectively $\mathfrak{g}_{0}$ ). As usual, let $\mathfrak{g}_{0}=\mathfrak{k}_{0} \oplus \mathfrak{p}_{0}$ be a Cartan decomposition of $\mathfrak{g}_{0}, \mathfrak{g}=\mathfrak{k} \oplus \mathfrak{p}$ its complexification, and $\theta$ the Cartan involution. Let $\sigma$ be the complex conjugation of $\mathfrak{g}$ with respect to $\mathfrak{g}_{0}$, and let $\mathfrak{h}$ be a $\sigma$-stable Cartan subalgebra of $\mathfrak{k}$. Since $\mathfrak{g}_{0}$ is of inner type, $\mathfrak{h}$ is also a Cartan subalgebra of $\mathfrak{g}$.

Denote by $\mathcal{N}$ the nilpotent variety of $\mathfrak{g}$ and set

$$
\mathcal{N}_{\mathbf{R}}=\mathcal{N} \cap \mathfrak{g}_{0}, \quad \mathcal{N}_{1}=\mathcal{N} \cap \mathfrak{p} .
$$

Let $K^{0}$ be the connected subgroup of $G$ with Lie algebra $\mathfrak{k}$. It is known that the orbit spaces $\mathcal{N}_{\mathbf{R}} / G_{0}$ and $\mathcal{N}_{1} / K^{0}$, equipped with the quotient topologies, are homeomorphic and that the Kostant-Sekiguchi bijection is a homeomorphism $\mathcal{N}_{\mathbf{R}} / G_{0} \rightarrow \mathcal{N}_{1} / K^{0}$ (see $[6,1])$. We can think of the closure diagram for adjoint nilpotent orbits in $\mathfrak{g}_{0}$ as describing the topology of $\mathcal{N}_{\mathbf{R}} / G_{0}$ (or, equivalently, $\mathcal{N}_{1} / K^{0}$ ).

Our main result is the closure diagram depicted in Figure 3. In order to construct this diagram and prove its correctness, it was necessary to perform extensive nontrivial computations. For this purpose, in addition to our own programs, we used heavily Maple [5] and, to a lesser extent, $\mathrm{LiE}$ [18].

2. Preliminaries. The closure diagram for adjoint nilpotent orbits in $\mathfrak{g}$ was determined by Mizuno [14] and verified later by Beynon and Spaltenstein [2]. We give this diagram in Figures 1 and 2 where each node represents a $G$-orbit in $\mathcal{N}$ and is labelled by the corresponding Bala-Carter symbol (see $[6,4])$. This diagram is a modified form of the one given in [17, p. 249]. In our diagram, the orbits having the same dimension are positioned at the same level. Because of its length, the diagram is split into three pieces. The bottom and the top portions of the diagram are shown in Figure 1, while the middle part is shown separately in Figure 2. The dimensions of the orbits are indicated on the left of the diagrams.

\footnotetext{
${ }^{*}$ Received October 24, 2000; accepted for publication November 13, 2000.

tDepartment of Pure Mathematics, University of Waterloo, Waterloo, Ontario, N2L 3G1, Canada (djokovic@uwaterloo.ca). Supported in part by the NSERC Grant A-5285.
} 
The line in the diagram [17, p. 249] joining the nodes $2 A_{4}$ and $D_{6}\left(a_{2}\right)$ is redundant as the diagram contains the lines joining $2 A_{4}$ to $A_{5}+A_{2}$ and the one joining $A_{5}+A_{2}$ to $D_{6}\left(a_{2}\right)$. (The Bala-Carter labels for the nodes $D_{6}\left(a_{2}\right), A_{5}+A_{2}, 2 A_{4}$ in $[17, \mathrm{p} .249]$ are $D_{6}\left(a_{2}\right), E_{7}\left(a_{5}\right), E_{8}\left(a_{7}\right)$, respectively.) Consequently, we have omitted that line from our diagram.

We remark that the closure diagram given in [4, p. 444] for this case has two errors: First, the line joining the nodes $A_{3}$ and $D_{4}\left(a_{1}\right)$ should be replaced by a line joining $A_{3}$ to $A_{3}+A_{1}$. Second, one should insert a new line joining the nodes $E_{8}\left(b_{6}\right)$ and $E_{6}+A_{1}$.

There are 70 adjoint nilpotent orbits in $\mathfrak{g}$ (including the trivial orbit). The nonzero ones are listed in Table 1 . The $k$-th orbit, i.e., the one that appears as the $k$-th entry in Table 1, will be denoted by $\mathcal{O}^{k}$. The second column of this table contains the BalaCarter symbol of $\mathcal{O}^{k}$, and the third one gives the weights of the weighted Dynkin diagram of $\mathcal{O}^{k}$. The complex dimension of $\mathcal{O}^{k}$ is recorded in the last column.

The nonzero $G_{0}$-orbits in $\mathcal{N}_{\mathbf{R}}$, or equivalently the nonzero $K^{0}$-orbits in $\mathcal{N}_{1}$, were classified in [7] (see also [6]). We shall keep the same numbering for these orbits as in these two references. The $i$-th nontrivial $G_{0}$-orbit in $\mathcal{N}_{\mathbf{R}}$ will be denoted by $\mathcal{O}_{0}^{i}$, and we denote by $\mathcal{O}_{1}^{i}$ the nontrivial $K^{0}$-orbit in $\mathcal{N}_{1}$ that corresponds to $\mathcal{O}_{0}^{i}$ under the Kostant-Sekiguchi bijection. In the fourth and fifth columns of Table 1 we list the superscripts $i$ of the orbits $\mathcal{O}_{0}^{i}$ (or, equivalently, $\mathcal{O}_{1}^{i}$ ) which are contained in $\mathcal{O}^{k}$. This depends on the type of the real form $\mathfrak{g}_{0}$ of $\mathfrak{g}$ (for the sake of completeness we have included also the split real form E VIII). For instance, if $k=4$ then:

$$
\begin{array}{lll}
\text { E VIII: } & \mathcal{O}^{4} \cap \mathfrak{g}_{0}=\mathcal{O}_{0}^{4} \cup \mathcal{O}_{0}^{5}, & \mathcal{O}^{4} \cap \mathfrak{p}=\mathcal{O}_{1}^{4} \cup \mathcal{O}_{1}^{5} \\
\text { E IX: } & \mathcal{O}^{4} \cap \mathfrak{g}_{0}=\mathcal{O}_{0}^{6} \cup \mathcal{O}_{0}^{7} \cup \mathcal{O}_{0}^{8}, & \mathcal{O}^{4} \cap \mathfrak{p}=\mathcal{O}_{1}^{6} \cup \mathcal{O}_{1}^{7} \cup \mathcal{O}_{1}^{8} .
\end{array}
$$

Recall that a triple $(E, H, F)$ in $\mathfrak{g}$ is called a standard triple if $[H, E]=2 E$, $[H, F]=-2 F,[F, E]=H$ and $E, H, F$ are nonzero. Such a triple is normal if also $H \in \mathfrak{k}$ and $E, F \in \mathfrak{p}$. We denote the root system of $(\mathfrak{g}, \mathfrak{h})$ by $R$, and choose a system of positive roots $R^{+} \subset R$ and a base $B=\left\{\alpha_{i}: 1 \leq i \leq 8\right\} \subset R^{+}$of $R$ (see Figure 4 in the Appendix). The simple roots $\alpha_{i} \in B$ are indexed as in [3].

Let us also introduce the subgroup $K=\{x \in G: \theta(x)=x\}$. Its identity component is the group $K^{0}$ defined above. In the case that concerns us here, namely E IX, we have $K=K^{0}$ (see [13]).

We extend the enumeration of simple roots $\alpha_{i}, 1 \leq i \leq 8$, to the enumeration $\alpha_{i}$, $1 \leq i \leq 120$, of $R^{+}$. It is the same as the one used in [8]. We have reproduced it in the Appendix. A negative root $-\alpha_{i}$ will be also written as $\alpha_{-i}$. The coroot of $\alpha_{i}$ is denoted by $H_{i} \in \mathfrak{h}$. Note that $H_{-i}=-H_{i}$. For $\alpha \in R$ we let $\mathfrak{g}^{\alpha}$ be the root space of $\alpha$. A nonzero element $X_{\alpha} \in \mathfrak{g}^{\alpha}$ is called a root vector of $\alpha$. We assume that a root 
vector $X_{i}$ is fixed for each root $\alpha_{i},|i| \in\{1, \ldots, 120\}$.

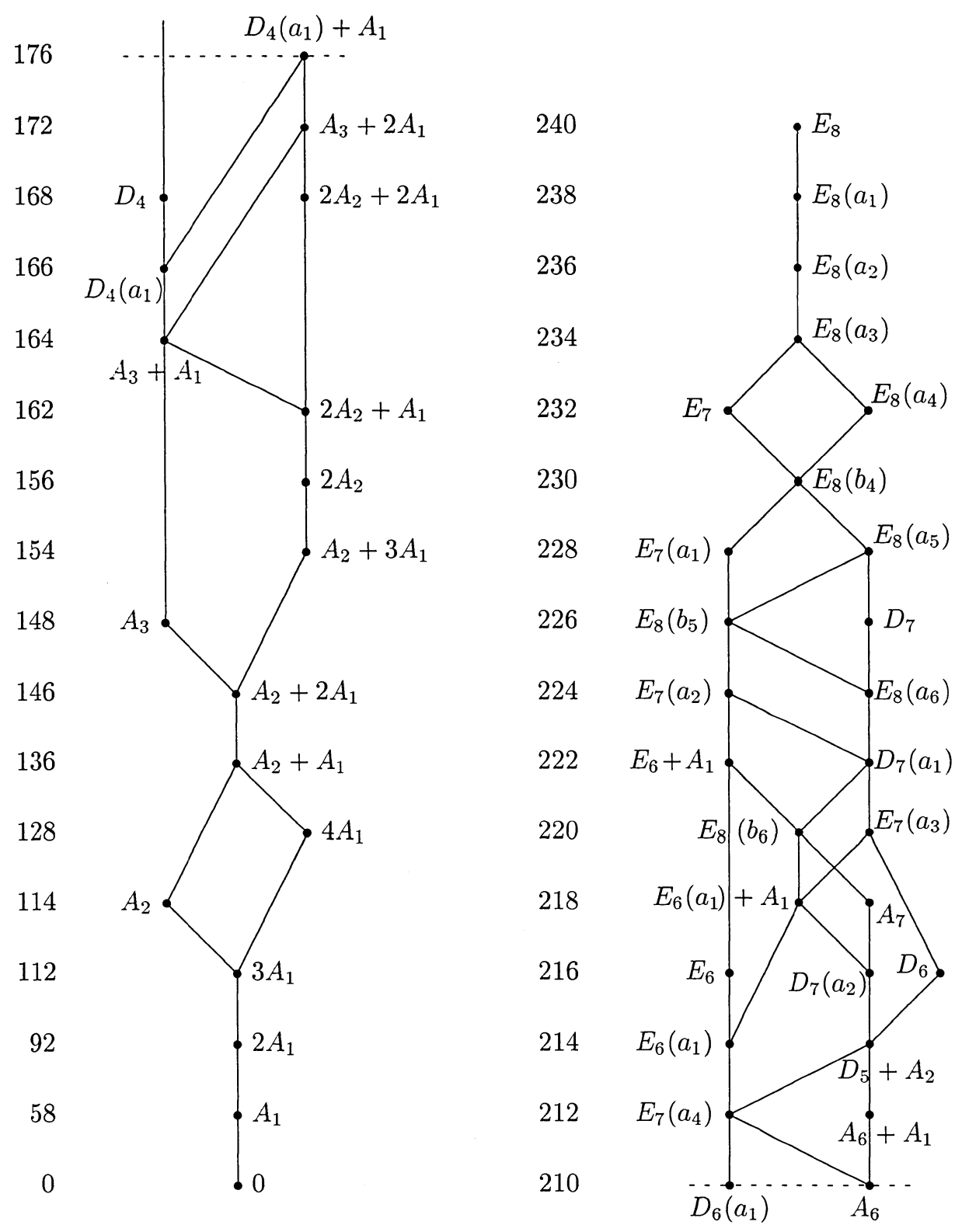

Figure 1: The bottom and top portions of the closure diagram of $E_{8}$ 


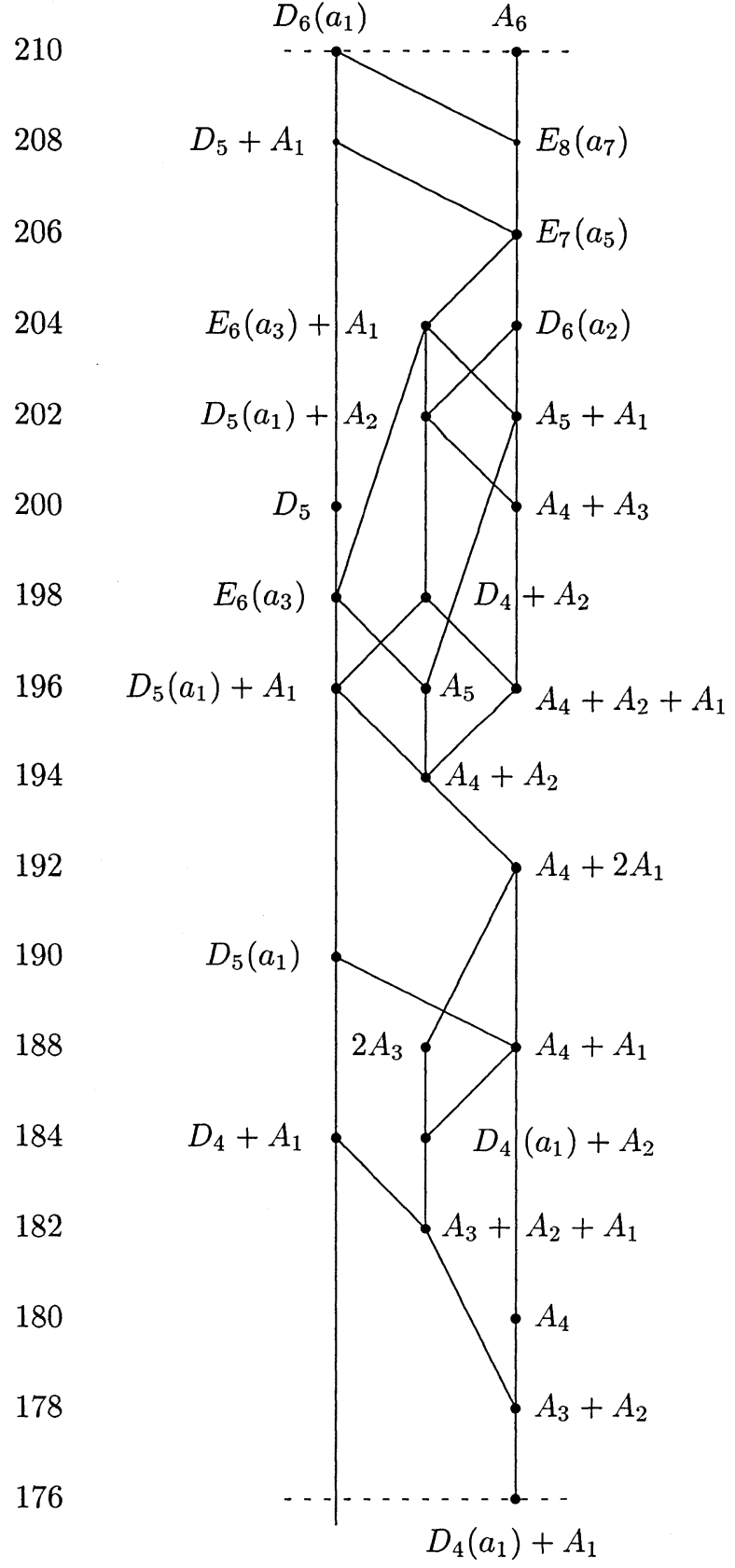

Figure 2: The middle part of the closure diagram of $E_{8}$ 
Table 1: Nonzero nilpotent adjoint orbits $\mathcal{O}^{k}$ in $\mathbf{E}_{\mathbf{8}}$

\begin{tabular}{|r|l|c|l|l|r|}
\hline$k$ & \multicolumn{1}{|c|}{ Label } & $\alpha_{j}(H)$ & E VIII & E IX & dim \\
\hline 1 & $A_{1}$ & 00000001 & 1 & 1 & 58 \\
2 & $2 A_{1}$ & 10000000 & 2 & 2,3 & 92 \\
3 & $3 A_{1}$ & 00000010 & 3 & 4,5 & 112 \\
4 & $A_{2}$ & 00000002 & 4,5 & $6,7,8$ & 114 \\
5 & $4 A_{1}$ & 01000000 & 6 & & 128 \\
6 & $A_{2}+A_{1}$ & 10000001 & 7,8 & 9 & 136 \\
7 & $A_{2}+2 A_{1}$ & 00000100 & 9,10 & 10,11 & 146 \\
8 & $A_{3}$ & 10000002 & 11 & 12,13 & 148 \\
9 & $A_{2}+3 A_{1}$ & 00100000 & 12,13 & & 154 \\
10 & $2 A_{2}$ & 20000000 & $14,15,16$ & 14 & 156 \\
11 & $2 A_{2}+A_{1}$ & 10000010 & 17 & 15 & 162 \\
12 & $A_{3}+A_{1}$ & 00000101 & 18 & 16,17 & 164 \\
13 & $D_{4}\left(a_{1}\right)$ & 00000020 & 19,20 & $18,19,20$ & 166 \\
14 & $D_{4}$ & 00000022 & 21 & 21,22 & 168 \\
15 & $2 A_{2}+2 A_{1}$ & 00001000 & 22 & & 168 \\
16 & $A_{3}+2 A_{1}$ & 00100001 & 23 & & 172 \\
17 & $D_{4}\left(a_{1}\right)+A_{1}$ & 01000010 & 24,25 & & 176 \\
18 & $A_{3}+A_{2}$ & 10000100 & $26,27,28$ & 23 & 178 \\
19 & $A_{4}$ & 20000002 & 29,30 & 24,25 & 180 \\
20 & $A_{3}+A_{2}+A_{1}$ & 00010000 & 31,32 & & 182 \\
21 & $D_{4}+A_{1}$ & 01000012 & 33 & & 184 \\
22 & $D_{4}\left(a_{1}\right)+A_{2}$ & 02000000 & $34,35,36$ & & 184 \\
23 & $A_{4}+A_{1}$ & 10000101 & 37,38 & 26 & 188 \\
24 & $2 A_{3}$ & 10001000 & 39 & & 188 \\
25 & $D_{5}\left(a_{1}\right)$ & 10000102 & 40,41 & 27 & 190 \\
26 & $A_{4}+2 A_{1}$ & 00010001 & $42,43,44$ & & 192 \\
27 & $A_{4}+A_{2}$ & 00000200 & 45,46 & 28 & 194 \\
28 & $A_{5}$ & 20000101 & 47 & 29 & 196 \\
29 & $D_{5}\left(a_{1}\right)+A_{1}$ & 00010002 & 48,49 & & 196 \\
30 & $A_{4}+A_{2}+A_{1}$ & 00100100 & 50 & & 196 \\
31 & $D_{4}+A_{2}$ & 02000002 & $51,52,53$ & & 198 \\
32 & $E_{6}\left(a_{3}\right)$ & 20000020 & $54,5.5$ & 30,31 & 198 \\
33 & $D_{5}$ & 20000022 & 56 & 32,33 & 200 \\
34 & $A_{4}+A_{3}$ & 00010010 & 57 & & 200 \\
35 & $A_{5}+A_{1}$ & 10010001 & 58 & & 202 \\
36 & $D_{5}\left(a_{1}\right)+A_{2}$ & 00100101 & 59 & & 202 \\
37 & $D_{6}\left(a_{2}\right)$ & 01100010 & 60,61 & & 204 \\
38 & $E_{6}\left(a_{3}\right)+A_{1}$ & 10001010 & 62,63 & & 204 \\
39 & $E_{7}\left(a_{5}\right)$ & 00010100 & 64,65 & & 206 \\
40 & $D_{5}+A_{1}$ & 10001012 & 66 & & 208 \\
41 & $E_{8}\left(a_{7}\right)$ & 00002000 & $67,68,69$ & & 208 \\
42 & $A_{6}$ & 20000200 & 70,71 & 34 & 210 \\
43 & $D_{6}\left(a_{1}\right)$ & 01100012 & 72,73 & & 210 \\
\hline
\end{tabular}


Table 1: (continued)

\begin{tabular}{|r|l|c|l|l|c|}
\hline$k$ & \multicolumn{1}{|c|}{ Label } & $\alpha_{j}(H)$ & E VIII & E IX & dim \\
\hline 44 & $A_{6}+A_{1}$ & 10010100 & 74 & & 212 \\
45 & $E_{7}\left(a_{4}\right)$ & 00010102 & 75,76 & & 212 \\
46 & $E_{6}\left(a_{1}\right)$ & 20000202 & 77,78 & 35 & 214 \\
47 & $D_{5}+A_{2}$ & 00002002 & $79,80,81$ & & 214 \\
48 & $E_{6}$ & 20000222 & 82 & 36 & 216 \\
49 & $D_{6}$ & 21100012 & 83 & & 216 \\
50 & $D_{7}\left(a_{2}\right)$ & 10010101 & 84,85 & & 216 \\
51 & $A_{7}$ & 10010110 & 86 & & 218 \\
52 & $E_{6}\left(a_{1}\right)+A_{1}$ & 10010102 & 87,88 & & 218 \\
53 & $E_{7}\left(a_{3}\right)$ & 20010102 & 89,90 & & 220 \\
54 & $E_{8}\left(b_{2}\right)$ & 00020002 & 91,92 & & 220 \\
55 & $D_{7}\left(a_{1}\right)$ & 20002002 & $93,94,95$ & & 222 \\
56 & $E_{6}+A_{1}$ & 10010122 & 96 & & 222 \\
57 & $E_{7}\left(a_{2}\right)$ & 01101022 & 97 & & 224 \\
58 & $E_{8}\left(a_{6}\right)$ & 00020020 & 98,99 & & 224 \\
59 & $D_{7}$ & 21101101 & 100 & & 226 \\
60 & $E_{8}\left(b_{5}\right)$ & 00020022 & 101,102 & & 226 \\
61 & $E_{7}\left(a_{1}\right)$ & 21101022 & 103 & & 228 \\
62 & $E_{8}\left(a_{5}\right)$ & 20020020 & 104,105 & & 228 \\
63 & $E_{8}\left(b_{4}\right)$ & 20020022 & 106,107 & & 230 \\
64 & $E_{7}$ & 21101222 & 108 & & 232 \\
65 & $E_{8}\left(a_{4}\right)$ & 20020202 & 109,110 & & 232 \\
66 & $E_{8}\left(a_{3}\right)$ & 20020222 & 111,112 & & 234 \\
67 & $E_{8}\left(a_{2}\right)$ & 22202022 & 113 & & 236 \\
68 & $E_{8}\left(a_{1}\right)$ & 22202222 & 114 & & 238 \\
69 & $E_{8}$ & 22222222 & 115 & & 240 \\
\hline
\end{tabular}

By adjoining the negative of the highest root: $\alpha_{0}=-\alpha_{120}=\alpha_{-120}$ to $B$ we obtain the so-called extended base $B_{e}=B \cup\left\{\alpha_{0}\right\}$. Let $R_{0}$ be the root system of $(\mathfrak{k}, \mathfrak{h})$ where we view $R_{0}$ as a subsystem of $R$. We set $R_{0}^{+}=R_{0} \cap R^{+}$and denote by $B_{0}$ the unique base of $R_{0}$ contained in $R_{0}^{+}$. It turns out that $B_{0} \subset B_{e}$. Explicitly we have:

$$
\begin{array}{ll}
\text { E VIII: } & B_{0}=\left\{\alpha_{0}, \alpha_{2}, \alpha_{3}, \alpha_{4}, \alpha_{5}, \alpha_{6}, \alpha_{7}, \alpha_{8}\right\}, \\
\text { E IX: } & B_{0}=\left\{\alpha_{0}, \alpha_{1}, \alpha_{2}, \alpha_{3}, \alpha_{4}, \alpha_{5}, \alpha_{6}, \alpha_{7}\right\} .
\end{array}
$$

Given a $K^{0}$-orbit $\mathcal{O}_{1}^{i} \subset \mathcal{N}_{1}$, we can choose a normal triple $(E, H, F)$ such that $E \in \mathcal{O}_{1}^{i}, H \in \mathfrak{h}$, and $\alpha(H) \geq 0$ for all $\alpha \in B_{0}$. The integers $\alpha(H)$ for $\alpha \in B_{0}$ determine uniquely $H$ and, consequently, also the orbit $\mathcal{O}_{1}^{i}$.

As in [7], in the case E IX we set (see Figure 4)

$$
\beta_{i}=\alpha_{i}, \quad(1 \leq i \leq 7) ; \quad \beta_{8}=\alpha_{0} .
$$

The technique developed in [9] to find the closure diagrams is especially convenient for real forms of inner type and will be employed in this paper. The closure diagram for the case EIX is given in Figure 3. 
Table 2: Representatives of the nonzero nilpotent $K$-orbits $\mathcal{O}_{1}^{i}$ in $\mathfrak{p}$

\begin{tabular}{|c|c|c|c|c|}
\hline$k$ & $i$ & $\overline{\beta_{j}\left(H^{i}\right)}$ & $E^{i}$ & Type \\
\hline 1 & 1 & 00000011 & $X_{-8}$ & $A_{1}$ \\
\hline 2 & 2 & 10000002 & $\left(X_{-8}\right)+\left(X_{-74}\right)$ & $2 A_{1}$ \\
\hline 2 & 3 & 00000100 & $\left(X_{118}\right)+\left(X_{-8}\right)$ & $2 A_{1}$ \\
\hline 3 & 4 & 00000013 & $\left(X_{-15}\right)+\left(X_{-68}\right)+\left(X_{-101}\right)$ & $3 A_{1}$ \\
\hline 3 & 5 & 10000011 & $\left(X_{119}\right)+\left(X_{-15}\right)+\left(X_{-68}\right)$ & $3 A_{1}$ \\
\hline 4 & 6 & 00000004 & $\begin{array}{l}X_{-8}+X_{-119} \\
\left(X_{-8}\right)+\left(X_{-74}\right)+\left(X_{-104}\right)+\left(X_{-118}\right)\end{array}$ & $\begin{array}{l}A_{2} \\
\left(4 A_{1}\right)^{\prime \prime}\end{array}$ \\
\hline 4 & 7 & 00000022 & $\left(X_{119}\right)+\left(X_{-15}\right)+\left(X_{-68}\right)+\left(X_{-101}\right)$ & $\left(4 A_{1}\right)^{\prime \prime}$ \\
\hline 4 & 8 & 20000000 & $\begin{array}{l}X_{101}+X_{-8} \\
\left(X_{101}\right)+\left(X_{119}\right)+\left(X_{-15}\right)+\left(X_{-68}\right)\end{array}$ & $\begin{array}{l}A_{2} \\
\left(4 A_{1}\right)^{\prime \prime}\end{array}$ \\
\hline 6 & 9 & 11000001 & $\left(X_{113}+X_{-42}\right)+\left(X_{-47}\right)$ & $A_{2}+A_{1}$ \\
\hline 7 & 10 & 10000102 & $\left(X_{118}+X_{-68}\right)+\left(X_{-47}\right)+\left(X_{-81}\right)$ & $A_{2}+2 A_{1}$ \\
\hline 7 & 11 & 00010000 & $\left(X_{110}+X_{-36}\right)+\left(X_{113}\right)+\left(X_{-50}\right)$ & $A_{2}+2 A_{1}$ \\
\hline 8 & 12 & 10000024 & $X_{119}+X_{-74}+X_{-101}$ & $A_{3}$ \\
\hline 8 & 13 & 00000122 & $X_{-68}+X_{118}+X_{-101}$ & $A_{3}$ \\
\hline 10 & 14 & 00000200 & $\left(X_{96}+X_{-22}\right)+\left(X_{112}+X_{-62}\right)$ & $2 A_{2}$ \\
\hline 11 & 15 & 10000111 & $\begin{array}{l}\left(X_{107}+X_{-47}\right)+\left(X_{117}+X_{-81}\right) \\
+\left(X_{-68}\right)\end{array}$ & $2 A_{2}+A_{1}$ \\
\hline 12 & 16 & 10000113 & $\left(X_{-73}+X_{118}+X_{-98}\right)+\left(X_{-74}\right)$ & $A_{3}+A_{1}$ \\
\hline 12 & 17 & 10000031 & $\left(X_{-47}+X_{104}+X_{-81}\right)+\left(X_{118}\right)$ & $A_{3}+A_{1}$ \\
\hline 13 & 18 & 00000204 & $\begin{array}{l}X_{118}+X_{-101}+X_{-68}+X_{-74} \\
\left(X_{-68}+X_{118}+X_{-101}\right)+\left(X_{-79}\right) \\
+\left(X_{-102}\right)\end{array}$ & $\begin{array}{l}D_{4}\left(a_{1}\right) \\
\left(A_{3}+2 A_{1}\right)^{\prime \prime}\end{array}$ \\
\hline 13 & 19 & 00000040 & $\begin{array}{l}X_{-15}+X_{74}+X_{104}+X_{101} \\
\left(X_{-22}+X_{74}+X_{-62}\right)+\left(X_{104}\right) \\
+\left(X_{118}\right) \\
\left(X_{74}+X_{-22}\right)+\left(X_{104}+X_{-67}\right) \\
+\left(X_{118}+X_{-98}\right)\end{array}$ & $\begin{array}{l}D_{4}\left(a_{1}\right) \\
\left(A_{3}+2 A_{1}\right)^{\prime \prime} \\
3 A_{2}\end{array}$ \\
\hline 13 & 20 & 20000022 & $\begin{array}{l}\left(X_{-47}+X_{104}+X_{-81}\right)+\left(X_{118}\right) \\
+\left(X_{-74}\right)\end{array}$ & $\left(A_{3}+2 A_{1}\right)^{\prime \prime}$ \\
\hline 14 & 21 & 00000048 & $X_{-74}+X_{119}+X_{-104}+X_{-118}$ & $D_{4}$ \\
\hline 14 & 22 & 20000044 & $X_{104}+X_{-101}+X_{118}+X_{-74}$ & $D_{4}$ \\
\hline 18 & 23 & 01100012 & $\begin{array}{l}\left(X_{-65}+X_{114}+X_{-87}\right) \\
+\left(X_{112}+X_{-74}\right)\end{array}$ & $A_{3}+A_{2}$ \\
\hline 19 & 24 & 40000004 & $\begin{array}{l}X_{101}+X_{-47}+X_{-112}+X_{119} \\
\left(X_{-47}+X_{101}+X_{-81}\right)+ \\
\left(X_{-100}+X_{119}+X_{-110}\right)\end{array}$ & $\begin{array}{l}A_{4} \\
2 A_{3}\end{array}$ \\
\hline 19 & 25 & 20000200 & $\begin{array}{l}X_{101}+X_{-47}+X_{96}+X_{-68} \\
\left(X_{96}+X_{-68}+X_{112}\right)+ \\
\left(X_{-54}+X_{101}+X_{-77}\right)\end{array}$ & $\begin{array}{l}A_{4} \\
2 A_{3}\end{array}$ \\
\hline 23 & 26 & 10100111 & $\begin{array}{l}\left(X_{104}+X_{-65}+X_{105}+X_{-74}\right) \\
+\left(X_{-73}\right)\end{array}$ & $A_{4}+A_{1}$ \\
\hline 25 & 27 & 01100034 & $X_{114}+X_{-98}+X_{-79}+X_{112}+X_{113}$ & $D_{5}\left(a_{1}\right)$ \\
\hline 27 & 28 & 00020000 & $\begin{array}{l}\left(X_{102}+X_{-56}+X_{94}+X_{-67}\right) \\
+\left(X_{107}+X_{-78}\right)\end{array}$ & $A_{4}+A_{2}$ \\
\hline 28 & 29 & 10000313 & $X_{-73}+X_{96}+X_{-74}+X_{112}+X_{-98}$ & $A_{5}$ \\
\hline
\end{tabular}


Table 2: (continued)

\begin{tabular}{|c|c|c|c|c|}
\hline$k$ & $i$ & $\beta_{j}\left(H^{i}\right)$ & $E^{i}$ & Type \\
\hline 32 & 30 & 20000222 & $\begin{array}{l}\left(X_{-73}+X_{96}+X_{-74}+X_{112}+X_{-98}\right) \\
+\left(X_{104}\right)\end{array}$ & $\left(A_{5}+A_{1}\right)^{\prime \prime}$ \\
\hline 32 & 31 & 00000404 & $\begin{array}{l}\left(X_{-79}+X_{96}+X_{-68}+X_{112}+X_{-102}\right) \\
+\left(X_{-101}\right) \\
X_{96}+X_{-74}+X_{112}+X_{114}+X_{-101} \\
+X_{113}\end{array}$ & $\begin{array}{l}\left(A_{5}+A_{1}\right)^{\prime \prime} \\
E_{6}\left(a_{3}\right)\end{array}$ \\
\hline 33 & 32 & 20000244 & $X_{104}+X_{-101}+X_{-74}+X_{96}+X_{112}$ & $D_{5}$ \\
\hline 33 & 33 & 40000048 & $X_{118}+X_{-101}+X_{104}+X_{-79}+X_{-102}$ & $D_{5}$ \\
\hline 42 & 34 & 00020200 & $\begin{array}{l}X_{102}+X_{-77}+X_{94}+X_{-68}+X_{96} \\
+X_{-79}\end{array}$ & $A_{6}$ \\
\hline 46 & 35 & 40000404 & $\begin{array}{l}X_{-84}+X_{96}+X_{-73}+X_{101}+X_{-98} \\
+X_{112}+X_{-99} \\
X_{96}+X_{-73}+X_{101}+X_{105}+X_{-102} \\
+X_{104}\end{array}$ & $\begin{array}{l}\left(A_{7}\right)^{\prime \prime} \\
E_{6}\left(a_{1}\right)\end{array}$ \\
\hline 48 & 36 & 40000448 & $\begin{array}{l}X_{96}+X_{-101}+X_{-79}+X_{104}+X_{-102} \\
+X_{112}\end{array}$ & $E_{6}$ \\
\hline
\end{tabular}

For any integer $j$ we define:

$$
\begin{aligned}
& \mathfrak{g}_{H}(0, j)=\{X \in \mathfrak{k}:[H, X]=j X\}, \\
& \mathfrak{g}_{H}(1, j)=\{X \in \mathfrak{p}:[H, X]=j X\},
\end{aligned}
$$

and

$$
\mathfrak{p}_{i}(H)=\sum_{j \geq i} \mathfrak{g}_{H}(1, j)
$$

Let $Q_{H}$ be the parabolic subgroup of $K^{0}$ with Lie algebra

$$
\mathfrak{q}_{H}=\sum_{j \geq 0} \mathfrak{g}_{H}(0, j) .
$$

3. Statement of the main result. Recall that $\mathfrak{g}_{0}$ is assumed to be of type E IX. Hence $K=\left(\mathrm{E}_{7} \times \mathrm{SL}_{2}\right) / Z_{2}$, where $\mathrm{E}_{7}$ is simply connected and $Z_{2}$ is the diagonal central subgroup of order 2. (By $Z_{k}$ we denote a cyclic group of order $k$.) There are exactly 36 nontrivial $K$-orbits in $\mathcal{N}_{1}$ : denoted by $\mathcal{O}_{1}^{i}, 1 \leq i \leq 36$. We choose a normal triple $\left(E^{i}, H^{i}, F^{i}\right)$ with $E^{i} \in \mathcal{O}_{1}^{i}, H^{i} \in \mathfrak{h}$, and such that $\beta_{j}\left(H^{i}\right) \geq 0$ for $1 \leq j \leq 8$. 
Table 3: Root spaces in $\mathfrak{g}_{H^{i}}(1,2)$ and $\mathfrak{p}_{3}\left(H^{i}\right)$

\begin{tabular}{|c|c|}
\hline$i$ & Indices of roots \\
\hline 1 & -8 \\
\hline 2 & $-8,-15,-22,-29,-36,-42,-43,-50,-56,-62,-68,-74$ \\
\hline 3 & $118,119,-8,-15$ \\
\hline 4 & $\begin{array}{l}-15,-22,-29,-36,-42,-43,-47,-50,-54,-56,-60,-62,-65,-67 \\
-68,-72,-73,-77,-78,-81,-83,-86,-87,-90,-94,-98,-101 ;-8\end{array}$ \\
\hline 5 & $119,-15,-22,-29,-36,-42,-43,-50,-56,-62,-68 ;-8$ \\
\hline 6 & $\begin{array}{l}-8,-15,-22,-29,-36,-42,-43,-47,-50,-54,-56,-60,-62,-65, \\
-67,-68,-72,-73,-74,-77,-78,-79,-81,-83,-84,-86,-87,-88, \\
-90,-91,-92,-94,-95,-96,-98,-99,-100,-101,-102,-103,-104, \\
-105,-106,-107,-108,-109,-110,-111,-112,-113,-114, \\
-115,-116,-117,-118,-119\end{array}$ \\
\hline 7 & $\begin{array}{l}119,-15,-22,-29,-36,-42,-43,-47,-50,-54,-56,-60,-62 \\
-65,-67,-68,-72,-73,-77,-78,-81,-83,-86,-87,-90,-94 \\
-98,-101 ;-8\end{array}$ \\
\hline 8 & $\begin{array}{l}101,104,107,109,111,113,114,115,116,117,118,119,-8,-15 \\
-22,-29,-36,-42,-43,-50,-56,-62,-68,-74\end{array}$ \\
\hline 9 & $\begin{array}{l}113,115,116,117,118,119,-42,-47,-50,-56,-62,-68,-74 \\
-8,-15,-22,-29,-36,-43\end{array}$ \\
\hline 10 & $\begin{array}{l}118,119,-47,-54,-60,-65,-67,-68,-72,-74,-77,-81 \\
-22,-29,-36,-42,-43,-50,-56,-62,-8,-15\end{array}$ \\
\hline 11 & $\begin{array}{l}110,111,112,113,114,115,-36,-42,-43,-47,-50,-54 \\
116,117,118,119,-8,-15,-22,-29\end{array}$ \\
\hline 12 & $\begin{array}{l}119,-74,-101 ;-47,-54,-60,-65,-67,-72,-73,-77,-78,-81 \\
-83,-86,-87,-90,-94,-98,-15,-22,-29,-36,-42,-43,-50 \\
-56,-62,-68,-8\end{array}$ \\
\hline 13 & $\begin{array}{l}118,-68,-73,-78,-83,-86,-87,-90,-94,-98,-101 ;-22,-29 \\
-36,-42,-43,-47,-50,-54,-56,-60,-62,-65,-67,-72,-77 \\
-81,119,-15,-8\end{array}$ \\
\hline 14 & $\begin{array}{l}96,100,103,105,106,107,108,109,110,111,112,113,114 \\
115,116,117,-22,-29,-36,-42,-43,-47,-50,-54,-56,-60 \\
-62,-65,-67,-72,-77,-81 ; 118,119,-8,-15\end{array}$ \\
\hline 15 & $\begin{array}{l}107,109,111,113,114,115,116,117,-47,-54,-60,-65,-67 \\
-68,-72,-77,-81 ; 118,-22,-29,-36,-42,-43,-50,-56 \\
-62,119,-15,-8\end{array}$ \\
\hline 16 & $\begin{array}{l}118,-73,-74,-78,-83,-86,-87,-90,-94,-98 ; 119,-47,-54 \\
-60,-65,-67,-68,-72,-77,-81,-22,-29,-36,-42,-43 \\
-50,-56,-62,-15,-8\end{array}$ \\
\hline 18 & $\begin{array}{l}104,107,109,111,113,114,115,116,117,118,-47,-54,-60 \\
-65,-67,-72,-73,-77,-78,-81,-83,-86,-87,-90,-94,-98 \\
-15,-22,-29,-36,-42,-43,-50,-56,-62,-68,119,-8 \\
118,119,-68,-73,-74,-78,-79,-83,-84,-86,-87,-88,-90, \\
-91,-92,-94,-95,-98,-99,-101,-102,-104 ;-22,-29,-36,-42, \\
-43,-47,-50,-54,-56,-60,-62,-65,-67,-72,-77,-81,-8,-15\end{array}$ \\
\hline
\end{tabular}




\section{Table 3: (continued)}

\begin{tabular}{|c|c|}
\hline$i$ & Indices of roots \\
\hline \multirow[t]{3}{*}{19} & $74,79,84,88,91,92,95,96,99,100,102,103,104,105,106$ \\
\hline & $107,108,109,110,111,112,113,114,115,116,117,118,-15,-22$ \\
\hline & $-29,-36,-42,-43,-47,-50,-54,-56,-60,-62,-65,-67,-68,-72$, \\
\hline & $-73,-77,-78,-81,-83,-86,-87,-90,-94,-98,-101 ; 119,-8$ \\
\hline \multirow[t]{2}{*}{20} & $104,107,109,111,113,114,115,116,117,118,-47,-54,-60$ \\
\hline & $\begin{array}{l}-65,-67,-72,-73,-74,-77,-78,-81,-83,-86,-87,-90,-94,-98 \\
119,-15,-22,-29,-36,-42,-43,-50,-56,-62,-68,-8\end{array}$ \\
\hline \multirow[t]{4}{*}{21} & $119,-74,-79,-84,-88,-91,-92,-95,-96,-99,-100,-102$ \\
\hline & $-103,-104,-105,-106,-107,-108,-109,-110,-111,-112,-113$, \\
\hline & $\begin{array}{l}-114,-115,-116,-117,-118 ;-15,-22,-29,-36,-42,-43,-47, \\
-50-54-56-61-62-65-67-68-72-73\end{array}$ \\
\hline & $\begin{array}{l}-50,-54,-56,-60,-62,-65,-67,-68,-72,-73 \\
-77,-78,-81,-83,-86,-87,-90,-94,-98,-101,-8\end{array}$ \\
\hline \multirow[t]{2}{*}{22} & $104,107,109,111,113,114,115,116,117,118,-74,-101 ;-47$ \\
\hline & $\begin{array}{l}-54,-60,-65,-67,-72,-73,-77,-78,-81,-83,-86,-87,-90,-94 \\
-98,119,-15,-22,-29,-36,-42,-43,-50,-56,-62,-68,-8\end{array}$ \\
\hline \multirow[t]{2}{*}{23} & $112,113,114,-65,-72,-74,-77,-78,-79,-83,-87 ; 115,116$ \\
\hline & $\begin{array}{l}117,118,-50,-54,-56,-60,-62,-67,-68,-73,119,-42 \\
-43,-47,-15,-22,-29,-36,-8\end{array}$ \\
\hline \multirow[t]{2}{*}{24} & $101,104,107,109,111,113,114,115,116,117,118,119,-47$ \\
\hline & $\begin{array}{l}-54,-60,-65,-67,-72,-73,-77,-78,-79,-81,-83,-84,-86, \\
-87,-88,-90,-91,-92,-94,-95,-96,-98,-99,-100,-102 \\
-103,-105,-106,-108,-110,-112 ;-8,-15,-22,-29 \\
-36,-42,-43,-50,-56,-62,-68,-74\end{array}$ \\
\hline 25 & $\begin{array}{l}96,100,101,103,104,105,106,108,110,112,-47,-54,-60 \\
-65,-67,-68,-72,-74,-77,-81 ; 107,109,111,113 \\
114,115,116,117,-22,-29,-36,-42,-43 \\
-50,-56,-62,118,119,-8,-15\end{array}$ \\
\hline 26 & $\begin{array}{l}104,105,108,110,112,-65,-72,-73,-74,-77,-81 ; 107,109 \\
111,113,-47,-54,-60,-67,-68,114,115,116,117,-43,-50 \\
-56,-62,118,-22,-29,-36,-42,119,-15,-8\end{array}$ \\
\hline 27 & $\begin{array}{l}112,113,114,-74,-79,-98,-101 ; 115,116,117,118,-81 \\
-86,-90,-94,-65,-72,-77,-78,-83,-87,-50,-54,-56,-60 \\
-62,-67,-68,-73,119,-42,-43,-47,-15,-22,-29,-36,-8\end{array}$ \\
\hline 28 & $\begin{array}{l}94,98,99,101,102,103,104,105,106,107,108,109,-56,-60 \\
-62,-65,-67,-68,-72,-73,-74,-78,-79,-84 ; 110,111,112 \\
113,114,115,-36,-42,-43,-47,-50,-54,116,117,118 \\
119,-8,-15,-22,-29\end{array}$ \\
\hline 29 & $\begin{array}{l}96,100,103,105,106,108,110,112,-73,-74,-78,-83,-86 \\
-87,-90,-94,-98 ; 107,109,111,113,114,115,116,117,-68 \\
-47,-54,-60,-65,-67,-72,-77,-81,118,-22,-29,-36 \\
-42,-43,-50,-56,-62,119,-15,-8\end{array}$ \\
\hline 30 & $\begin{array}{l}96,100,103,104,105,106,108,110,112,-73,-74,-78,-83 \\
-86,-87,-90,-94,-98 ; 107,109,111,113,114,115,116,117 \\
-47,-54,-60,-65,-67,-68,-72,-77,-81,118,-22,-29 \\
-36,-42,-43,-50,-56,-62,119,-15,-8\end{array}$ \\
\hline
\end{tabular}


Table 3: (continued)

\begin{tabular}{|c|c|}
\hline$i$ & Indices of roots \\
\hline 31 & $\begin{array}{l}96,100,103,105,106,107,108,109,110,111,112,113,114, \\
115,116,117,-68,-73,-74,-78,-79,-83,-84,-86,-87,-88, \\
-90,-91,-92,-94,-95,-98,-99,-101,-102,-104 ; 118, \\
119,-22,-29,-36,-42,-43,-47,-50,-54,-56,-60,-62, \\
-65,-67,-72,-77,-81,-8,-15\end{array}$ \\
\hline 32 & $\begin{array}{l}96,100,103,104,105,106,108,110,112,-74,-101 ; 107 \\
109,111,113,114,115,116,117,-73,-78,-83,-86,-87,-90 \\
-94,-98,118,-47,-54,-60,-65,-67,-68,-72,-77,-81 \\
-22,-29,-36,-42,-43,-50,-56,-62,119,-15,-8\end{array}$ \\
\hline 33 & $\begin{array}{l}104,107,109,111,113,114,115,116,117,118,-79,-84,-88, \\
-91,-92,-95,-96,-99,-100,-101,-102,-103,-105,-106,-108, \\
-110,-112 ; 119,-47,-54,-60,-65,-67,-72,-73,-74, \\
-77,-78,-81,-83,-86,-87,-90,-94,-98,-15,-22,-29, \\
-36,-42,-43,-50,-56,-62,-68,-8\end{array}$ \\
\hline 34 & $\begin{array}{l}94,96,98,99,100,101,102,104,-68,-73,-74,-77,-78,-79 \\
-81,-84 ; 103,105,106,107,108,109,-56,-60,-62,-65,-67 \\
-72,110,111,112,113,114,115,-36,-42,-43,-47,-50 \\
-54,116,117,-22,-29,118,119,-8,-15\end{array}$ \\
\hline 35 & $\begin{array}{l}96,100,101,103,104,105,106,108,110,112,-73,-78,-79 \\
-83,-84,-86,-87,-88,-90,-91,-92,-94,-95,-98,-99,-102 \\
107,109,111,113,114,115,116,117,-47,-54,-60,-65,-67 \\
-68,-72,-74,-77,-81,118,119,-22,-29,-36,-42 \\
-43,-50,-56,-62,-8,-15\end{array}$ \\
\hline 36 & $\begin{array}{l}96,100,103,104,105,106,108,110,112,-79,-84,-88,-91, \\
-92,-95,-99,-101,-102 ; 107,109,111,113,114,115,116, \\
117,-73,-74,-78,-83,-86,-87,-90,-94,-98,118,-47,-54, \\
-60,-65,-67,-68,-72,-77,-81,119,-22,-29,-36,-42, \\
-43,-50,-56,-62,-15,-8\end{array}$ \\
\hline
\end{tabular}

These $36 K$-orbits are listed in Table 2 . For each $i \in\{1, \ldots, 36\}$ we record in the first column the integer $k$ for which $\mathcal{O}_{1}^{i} \subset \mathcal{O}^{k}$. In the third column we list the integers $\beta_{j}\left(H^{i}\right), 1 \leq j \leq 8$. They determine uniquely the element $H^{i} \in \mathfrak{h}$. Since $\mathfrak{k}$ is of type $E_{7}+A_{1}$ and $\left\{\beta_{i}: 1 \leq i \leq 7\right\}$ is a base for this $E_{7}$, we separate the last integer $\beta_{8}\left(H^{i}\right)$ from the first seven. In the fourth column we give a representative $E^{i} \in \mathcal{O}_{1}^{i}$. In each case, $E^{i} \in \mathfrak{g}_{H^{i}}(1,2)$ and $E^{i}$ is a sum of root vectors. The last column gives the type of the representative $E^{i}$ (to be defined below). In some cases we give several representatives of different types.

A subalgebra of $\mathfrak{g}$ is called regular if it is normalized by a Cartan subalgebra of $\mathfrak{g}$. We shall say that a regular subalgebra of $\mathfrak{g}$ is standard if it is normalized by $\mathfrak{h}$. Of course, every regular subalgebra is $G$-conjugate to a standard one. Most of the time, two isomorphic regular semisimple subalgebras are $G$-conjugate but there are 5 exceptions: 
In each of the exceptional cases there are two $G$-conjugacy classes, say $\left(4 A_{1}\right)^{\prime}$ and $\left(4 A_{1}\right)^{\prime \prime}$, and similarly in other cases. As mentioned in [8], the subalgebras $(X)^{\prime}$ are Levi subalgebras while $(X)^{\prime \prime}$ are not.
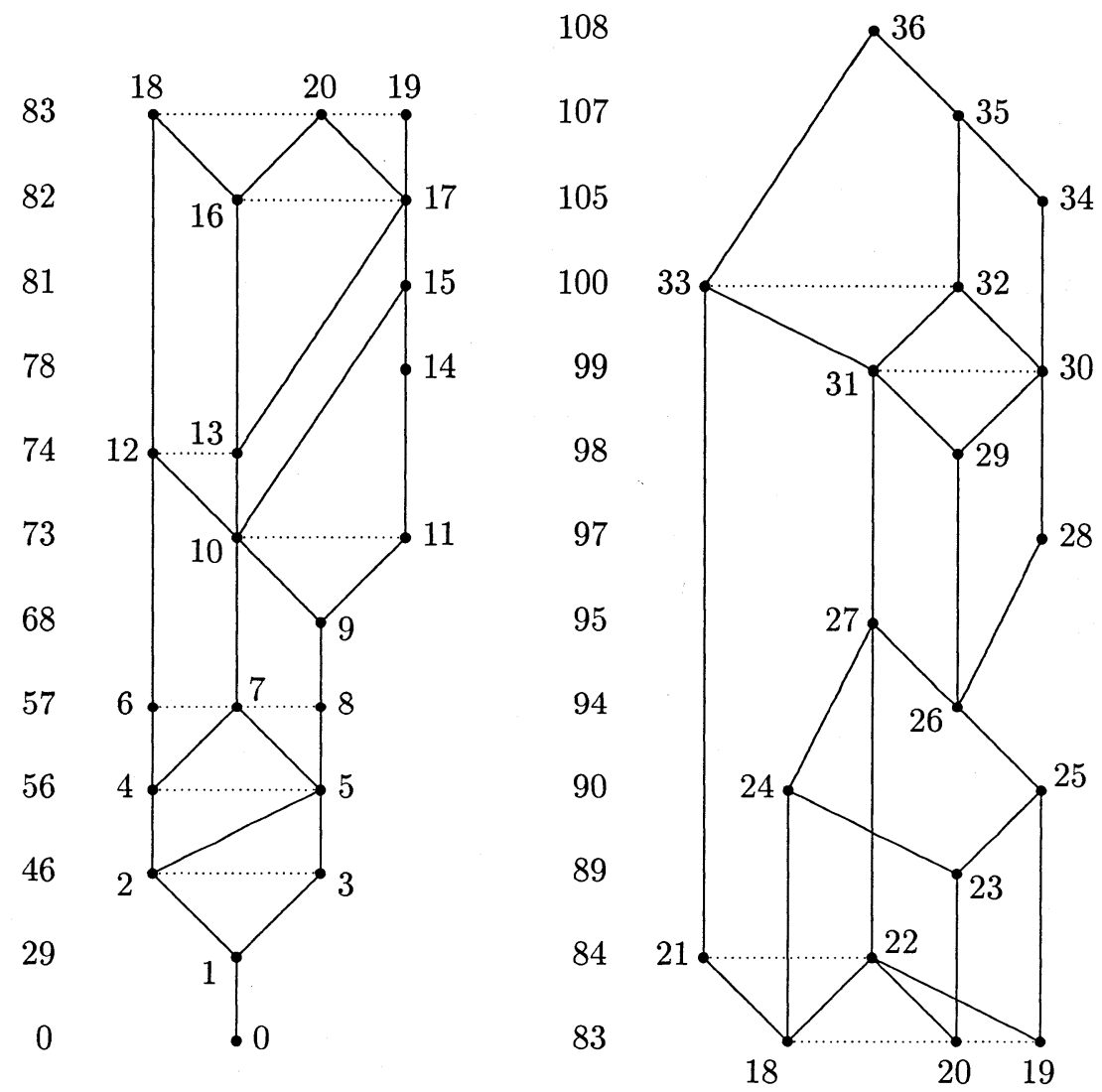

Figure 3: The closure diagram of adjoint nilpotent orbits of E IX

In most cases, the representative $E^{i} \in \mathcal{O}_{1}^{i}$ is the sum of root vectors for simple roots of a standard regular semisimple subalgebra and the type of $E^{i}$ is, by definition, the type of that subalgebra (up to $G$-conjugacy). If this is not the case, then the type of $E^{i}$ is the Bala-Carter symbol of the orbit $\mathcal{O}^{k}$ containing $\mathcal{O}_{1}^{i}$.

In Table 3 we list, for each $i$, the indices $k$ of the roots $\alpha=\alpha_{k}$ for which $\mathfrak{g}^{\alpha} \subset$ $\mathfrak{p}_{2}\left(H^{i}\right)$. We list first those indices for which $\mathfrak{g}^{\alpha} \subset \mathfrak{g}_{H^{i}}(1,2)$ and separate them by a semi-colon from the indices for which $\mathfrak{g}^{\alpha} \subset \mathfrak{p}_{3}\left(H^{i}\right)$.

THEOREM 3.1. Let $\mathfrak{g}_{0}$ be of type EIX. Then the closure ordering of the nilpotent $K$-orbits in $\mathfrak{p}$ is as given in Figure 3.

The horizontal dotted lines indicate that the $K$-orbits joined by these lines are contained in the same $G$-orbit. The numbers on the left hand side of the diagram are the complex dimensions of the orbits on that level. 
Table 4: Elements $E \in \mathfrak{p}_{2}\left(H^{i}\right) \cap \mathcal{O}_{1}^{j}$

\begin{tabular}{|c|c|c|c|}
\hline$i$ & $j$ & Type & $E$ \\
\hline 2,3 & 1 & $A_{1}$ & $X_{-8}$ \\
\hline 4,5 & 2 & $2 A_{1}$ & $\left(X_{-15}\right)+\left(X_{-68}\right)$ \\
\hline 5 & 3 & $2 A_{1}$ & $\left(X_{119}\right)+\left(X_{-15}\right)$ \\
\hline 6,7 & 4 & $3 A_{1}$ & $\left(X_{-15}\right)+\left(X_{-68}\right)+\left(X_{-101}\right)$ \\
\hline 7,8 & 5 & $3 A_{1}$ & $\left(X_{119}\right)+\left(X_{-15}\right)+\left(X_{-68}\right)$ \\
\hline 12 & 6 & $A_{2}$ & $X_{-74}+X_{-101}$ \\
\hline 10 & 7 & $\left(4 A_{1}\right)^{\prime \prime}$ & $\left(X_{119}\right)+\left(X_{-47}\right)+\left(X_{-68}\right)+\left(X_{-81}\right)$ \\
\hline 9 & 8 & $A_{2}$ & $X_{113}+X_{-42}$ \\
\hline 10 & 9 & $A_{2}+A_{1}$ & $\left(X_{118}+X_{-68}\right)+\left(X_{-47}\right)$ \\
\hline 11 & 9 & $A_{2}+A_{1}$ & $\left(X_{113}+X_{-42}\right)+\left(X_{-47}\right)$ \\
\hline 12 & 10 & $A_{2}+2 A_{1}$ & $\left(X_{119}+X_{-74}\right)+\left(X_{-47}\right)+\left(X_{-81}\right)$ \\
\hline 13,15 & 10 & $A_{2}+2 A_{1}$ & $\left(X_{118}+X_{-68}\right)+\left(X_{-47}\right)+\left(X_{-81}\right)$ \\
\hline 14 & 11 & $A_{2}+2 A_{1}$ & $\left(X_{114}+X_{-47}\right)+\left(X_{113}\right)+\left(X_{-50}\right)$ \\
\hline 18 & 12 & $A_{3}$ & $X_{119}+X_{-74}+X_{-101}$ \\
\hline 16,17 & 13 & $A_{3}$ & $X_{-86}+X_{118}+X_{-87}$ \\
\hline 15 & 14 & $2 A_{2}$ & $\left(X_{113}+X_{-67}\right)+\left(X_{114}+X_{-65}\right)$ \\
\hline 17 & 15 & $2 A_{2}+A_{1}$ & $\left(X_{113}+X_{-67}\right)+\left(X_{114}+X_{-65}\right)+\left(X_{-68}\right)$ \\
\hline 18,20 & 16 & $A_{3}+A_{1}$ & $\left(X_{-86}+X_{118}+X_{-87}\right)+\left(X_{-74}\right)$ \\
\hline 19,20 & 17 & $A_{3}+A_{1}$ & $\left(X_{-77}+X_{114}+X_{-78}\right)+\left(X_{113}\right)$ \\
\hline 21 & 18 & $\left(A_{3}+2 A_{1}\right)^{\prime \prime}$ & $\left(X_{-88}+X_{119}+X_{-95}\right)+\left(X_{-86}\right)+\left(X_{-87}\right)$ \\
\hline 22 & 18 & $D_{4}\left(a_{1}\right)$ & $X_{118}+X_{-101}+X_{-68}+X_{-74}$ \\
\hline 24 & 18 & $\left(A_{3}+2 A_{1}\right)^{\prime \prime}$ & $\left(X_{-73}+X_{118}+X_{-98}\right)+\left(X_{-84}\right)+\left(X_{-99}\right)$ \\
\hline 22 & 19 & $\left(A_{3}+2 A_{1}\right)^{\prime \prime}$ & $\left(X_{113}+X_{-101}+X_{114}\right)+\left(X_{-36}\right)+\left(X_{-50}\right)$ \\
\hline 25 & 19 & $\left(A_{3}+2 A_{1}\right)^{\prime \prime}$ & $\left(X_{-54}+X_{104}+X_{-77}\right)+\left(X_{96}\right)+\left(X_{112}\right)$ \\
\hline 22,23 & 20 & $\left(A_{3}+2 A_{1}\right)^{\prime \prime}$ & $\left(X_{-77}+X_{114}+X_{-78}\right)+\left(X_{113}\right)+\left(X_{-74}\right)$ \\
\hline 33 & 21 & $D_{4}$ & $X_{-79}+X_{104}+X_{-101}+X_{-102}$ \\
\hline 27 & 22 & $D_{4}$ & $X_{113}+X_{-101}+X_{114}+X_{-74}$ \\
\hline 24 & 23 & $A_{3}+A_{2}$ & $\left(X_{-65}+X_{114}+X_{-87}\right)+\left(X_{113}+X_{-79}\right)$ \\
\hline 25 & 23 & $A_{3}+A_{2}$ & $\left(X_{96}+X_{-68}+X_{112}\right)+\left(X_{101}+X_{-54}\right)$ \\
\hline 27 & 24 & $A_{4}$ & $X_{113}+X_{-79}+X_{-98}+X_{114}$ \\
\hline 26 & 25 & $A_{4}$ & $X_{104}+X_{-65}+X_{105}+X_{-74}$ \\
\hline 27 & 26 & $A_{4}+A_{1}$ & $\left(X_{114}+X_{-98}+X_{112}+X_{-74}\right)+\left(X_{-73}\right)$ \\
\hline 28 & 26 & $A_{4}+A_{1}$ & $\left(X_{104}+X_{-65}+X_{105}+X_{-74}\right)+\left(X_{-73}\right)$ \\
\hline 29 & 26 & $A_{4}+A_{1}$ & $\left(X_{112}+X_{-74}+X_{96}+X_{-83}\right)+\left(X_{-54}\right)$ \\
\hline 31 & 27 & $D_{5}\left(a_{1}\right)$ & $X_{114}+X_{-98}+X_{-79}+X_{112}+X_{113}$ \\
\hline 30 & 28 & $A_{4}+A_{2}$ & $\left(X_{108}+X_{-74}+X_{103}+X_{-73}\right)+\left(X_{104}+X_{-65}\right)$ \\
\hline 30,31 & 29 & $A_{5}$ & $X_{-73}+X_{96}+X_{-74}+X_{112}+X_{-98}$ \\
\hline 32 & 30 & $\left(A_{5}+A_{1}\right)^{\prime \prime}$ & $\left(X_{-73}+X_{96}+X_{-74}+X_{112}+X_{-98}\right)+\left(X_{104}\right)$ \\
\hline 34 & 30 & $\left(A_{5}+A_{1}\right)^{\prime \prime}$ & $\left(X_{96}+X_{-68}+X_{94}+X_{-77}+X_{102}\right)+\left(X_{-54}\right)$ \\
\hline 32 & 31 & $E_{6}\left(a_{3}\right)$ & $X_{96}+X_{-74}+X_{112}+X_{114}+X_{-101}+X_{113}$ \\
\hline 33 & 31 & $\left(A_{5}+A_{1}\right)^{\prime \prime}$ & $\left(X_{-79}+X_{107}+X_{-101}+X_{117}+X_{-102}\right)+\left(X_{-68}\right)$ \\
\hline 35 & 32 & $D_{5}$ & $X_{112}+X_{-102}+X_{-73}+X_{96}+X_{101}$ \\
\hline 36 & 33 & $D_{5}$ & $X_{118}+X_{-101}+X_{104}+X_{-79}+X_{-102}$ \\
\hline 35 & 34 & $A_{6}$ & $X_{112}+X_{-98}+X_{101}+X_{-73}+X_{96}+X_{-84}$ \\
\hline 36 & 35 & $\left(A_{7}\right)^{\prime \prime}$ & $X_{-73}+X_{96}+X_{-84}+X_{104}+X_{-99}+X_{112}+X_{-98}$ \\
\hline
\end{tabular}


4. Proof of the main result. Let $i, j$ be a pair of nodes in the diagram of Figure 3, with $i$ above $j$, which are joined by a solid line. We prove that $\mathcal{O}_{1}^{i}>\mathcal{O}_{1}^{j}$ by showing that $\mathcal{O}_{1}^{j}$ meets $\mathfrak{p}_{2}\left(H^{i}\right)$ (see [9, Theorem 3.1]). In Table 4 we list all such pairs $i, j$ (with $j \neq 0$ ) and for each of them provide an element $E \in \mathfrak{p}_{2}\left(H^{i}\right) \cap \mathcal{O}_{1}^{j}$. We also indicate the type of $E$.

The fact that $E \in \mathfrak{p}_{2}\left(H^{i}\right)$ can be checked by using Table 3 . In most cases the verification of the claim that $E \in \mathcal{O}_{1}^{j}$ is straightforward: This element belongs to $\mathfrak{g}_{H^{j}}(1,2)$ and, being of the right type, is in fact a generic element for the action of the centralizer $Z_{K}\left(H^{j}\right)$. This argument is not applicable when $(i, j)$ is one of the following pairs:

$$
\begin{array}{llll}
(33,21), & (25,23), & (27,26), & (29,26), \\
(34,30), & (35,32), & (35,34) . &
\end{array}
$$

Since $\mathcal{O}^{18} \cap \mathfrak{p}=\mathcal{O}_{1}^{23}$ (see Table 1 ), we conclude that in the case $(25,23)$ we must have $E \in \mathcal{O}_{1}^{23}$. A similar argument is applicable to the pairs $(27,26),(29,26)$, and $(35,34)$. The remaining three pairs $(33,21),(34,30)$, and $(35,32)$ require a more elaborate argument.

Let us consider in detail the pair $(33,21)$. In this case

$$
E=X_{-79}+X_{104}+X_{-101}+X_{-102}
$$

is a standard principal nilpotent element of type $D_{4}$. Hence

$$
E \in \mathcal{O}^{14} \cap \mathfrak{p}=\mathcal{O}_{1}^{21} \cup \mathcal{O}_{1}^{22}
$$

and we have to show that in fact $E \in \mathcal{O}_{1}^{21}$. We do this by finding a normal triple $(E, H, F)$ inside the standard regular simple subalgebra of type $D_{4}$ having $\left\{\alpha_{-79}, \alpha_{104}\right.$, $\left.\alpha_{-101}, \alpha_{-102}\right\}$ as a base for its root system. The element $H$ is given by

$$
\begin{aligned}
H & =6 H_{-79}+10 H_{104}+6 H_{-101}+6 H_{-102} \\
& =-2\left(2 H_{1}+5 H_{2}+6 H_{3}+10 H_{4}+9 H_{5}+8 H_{6}+5 H_{7}+4 H_{8}\right) .
\end{aligned}
$$

We do not need to compute $F$ but we remark that

$$
F \in\left\langle X_{79}, X_{-104}, X_{101}, X_{102}\right\rangle \text {. }
$$

Next we compute

$$
\alpha_{m}(H)=4,0,0,0,0,-4,4,-6 \quad(1 \leq m \leq 8)
$$

and deduce that

$$
\beta_{m}(H)=4,0,0,0,0,-4,4,-6 \quad(1 \leq m \leq 8) .
$$

Finally by applying a suitable element $w$ of the Weyl group of $(\mathfrak{k}, \mathfrak{h})$ to $H$ we obtain the element $H^{\prime}=w(H)$ such that

$$
\beta_{m}\left(H^{\prime}\right)=0,0,0,0,0,0,4,8 \quad(1 \leq m \leq 8) .
$$


By looking up Table 2, we conclude that indeed $E \in \mathcal{O}_{1}^{21}$.

The argument is similar in the other two cases. We only state that for the pair $(34,30)$ we have

$$
\alpha_{m}(H)=2,2,-4,2,0,0,4,-8 \quad(1 \leq m \leq 8)
$$

and for $(35,32)$

$$
\alpha_{m}(H)=-2,-2,4,0,0,6,-6,8 \quad(1 \leq m \leq 8) .
$$

By inspection of Figure 3, we see that in order to complete the proof of the theorem it suffices to show that $\mathcal{O}_{1}^{i} \ngtr \mathcal{O}_{1}^{j}$ when $(i, j)$ is one of the following pairs:

$$
\begin{array}{lll}
(6,3), & (14,4), & (34,6), \\
(21,11), & (15,13), & (19,16), \\
(24,19), & (35,21), & (33,28) .
\end{array}
$$

This assertion is valid for the pair $(15,13)$ because $\mathcal{O}_{1}^{15} \subset \mathcal{O}^{11}, \mathcal{O}_{1}^{13} \subset \mathcal{O}^{8}$, and $\mathcal{O}^{11} \ngtr \mathcal{O}^{8}$ (see Table 1 and Figure 1). Another proof of this fact will be given below.

Let $V=\mathfrak{g}$ be the adjoint $\mathfrak{g}$-module (of dimension 248). It can be equipped with the $Z_{2}$-grading $V=V_{0} \oplus V_{1}$, where $V_{0}=\mathfrak{k}$ and $V_{1}=\mathfrak{p}$. Thus $\operatorname{dim} V_{0}=136$, and $\operatorname{dim} V_{1}=112$. We introduce the integers

$$
d_{i}(j, k)=\operatorname{dim} V_{i} \cap \operatorname{ker} \rho\left(E^{k}\right)^{j}
$$

where $i=0,1 ; j \geq 1 ; 1 \leq k \leq 36$, and $\rho=$ ad is the adjoint representation of $\mathfrak{g}$ on $V$. They are easy to compute and are displayed in Table 5.

By applying [9, Theorem 4.1] and using Table 5, we see that $\mathcal{O}_{1}^{i} \ngtr \mathcal{O}_{1}^{j}$ when $(i, j)$ is one of the following pairs.

$$
\begin{array}{lllll}
(6,3), & (11,4), & (6,5), & (23,6), & (12,11), \\
(19,12), & (23,12), & (15,13), & (19,16), & (18,17), \\
(23,19), & (21,20), & (31,21), & (34,21), & (24,22), \\
(28,22), & (28,24), & (34,32), & (34,33) . &
\end{array}
$$

In particular this means that the pairs $(6,3),(15,13)$, and $(19,16)$ from $(4.1)$ have been taken care of.

The remaining six pairs in the list (4.1) are handled by using the theory of prehomogeneous vector spaces (PV) $[15,16]$. 
Table 5: The integers $d_{i}(j, k)$ for the adjoint module of $E_{8}$ equipped with a $\mathrm{Z}_{2}$-gradation

\begin{tabular}{|c|c|c|}
\hline$k$ & $d_{0}(j, k) ; j=1,2, \ldots$ & $d_{1}(j, k) ; j=1,2,$. \\
\hline 1 & 107136 & 83111112 \\
\hline 2 & 90134136 & 66100112 \\
\hline 3 & 90126136 & 66108112 \\
\hline 4 & 80134135136 & 5683111112 \\
\hline 5 & 80118135136 & 5699111112 \\
\hline 6 & 79134135135136 & 5556111112 \\
\hline 7 & 79108135136 & 5582111111112 \\
\hline 8 & $79102 \quad 135135136$ & 5588111112 \\
\hline 9 & 68102129135136 & 4487105112 \\
\hline 10 & 63100125135136 & 3980101110112 \\
\hline 11 & $63 \quad 96125133136$ & 3984101112 \\
\hline 12 & 6290107134135136 & 385583100111111112 \\
\hline 13 & $6282107 \quad 126135136$ & 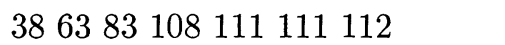 \\
\hline 14 & 5884122126136 & 347298108112 \\
\hline 15 & 5584115126135136 & 317191108111112 \\
\hline 16 & $5482 \quad 107 \quad 126 \quad 134136$ & $\begin{array}{lllllll}30 & 62 & 83 & 100 & 110 & 111 & 112\end{array}$ \\
\hline 17 & $5482 \quad 107118 \quad 134136$ & $\begin{array}{lllllll}30 & 62 & 83 & 108 & 110 & 111 & 112\end{array}$ \\
\hline 18 & $53 \quad 82107 \quad 125134136$ & $29548392 \quad 110110112$ \\
\hline 19 & $5382 \quad 107109134136$ & $295483 \quad 108 \quad 110110112$ \\
\hline 20 & $5375107 \quad 118134135136$ & 29618399110111112 \\
\hline 21 & $\begin{array}{l}528080107 \quad 107 \quad 134134135 \\
135136\end{array}$ & $\begin{array}{l}282856568383110110111 \\
111112\end{array}$ \\
\hline 22 & $\begin{array}{l}52648091107118134135 \\
135136\end{array}$ & $\begin{array}{l}284456728399110110111 \\
111112\end{array}$ \\
\hline 23 & 4770102117129134136 & 23597898105111112 \\
\hline 24 & $46 \quad 6890101 \quad 123 \quad 134135135136$ & 2244668899100111112 \\
\hline 25 & $46 \quad 6090101 \quad 123 \quad 126 \quad 135135136$ & 2252668899108111112 \\
\hline 26 & 426089101120126134135136 & 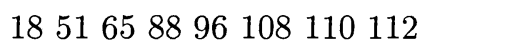 \\
\hline 27 & $\begin{array}{l}41588091 \quad 107118129134 \\
135136\end{array}$ & $\begin{array}{l}174356728398105110111 \\
111 \quad 112\end{array}$ \\
\hline 28 & $39548796 \quad 117 \quad 122 \quad 133133136$ & 1548638493104109112 \\
\hline 29 & $\begin{array}{l}38507384106116124126 \\
134136\end{array}$ & $\begin{array}{l}143749728297100108110 \\
111 \quad 112\end{array}$ \\
\hline 30 & $\begin{array}{l}37507384106109124126 \\
134135136\end{array}$ & $\begin{array}{l}133649718297100108110 \\
111112\end{array}$ \\
\hline 31 & $\begin{array}{l}37507383106116124126 \\
134136\end{array}$ & $\begin{array}{l}133649728290100108110 \\
110112\end{array}$ \\
\hline 32 & $\begin{array}{l}36 \quad 4863 \quad 748992 \quad 107 \quad 109 \quad 124 \\
126 \quad 134 \quad 135 \quad 135 \quad 136\end{array}$ & $\begin{array}{l}1227395465808398100108 \\
110110111 \quad 111 \quad 112\end{array}$ \\
\hline 33 & $\begin{array}{l}3648 \quad 63 \quad 74 \quad 89 \quad 100 \quad 107 \quad 117 \quad 124 \\
134 \quad 134 \quad 135 \quad 135 \quad 136\end{array}$ & $\begin{array}{l}1227395465728390100100 \\
110110111111112\end{array}$ \\
\hline 34 & $\begin{array}{l}313863699096114115125 \\
126133133136\end{array}$ & $\begin{array}{l}7323960668490100101108 \\
109112\end{array}$ \\
\hline 35 & 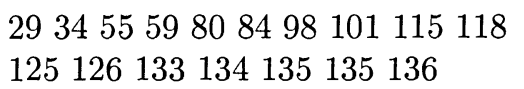 & $\begin{array}{l}5263152567074889198101 \\
108109110111 \quad 112\end{array}$ \\
\hline
\end{tabular}


Table 5: (continued)

\begin{tabular}{|c|l|l|}
\hline$k$ & \multicolumn{1}{|c|}{$d_{0}(j, k) ; j=1,2, \ldots$} & \multicolumn{1}{|c|}{$d_{1}(j, k) ; j=1,2, \ldots$} \\
\hline 36 & $\begin{array}{l}283246496366808397100 \\
\\
107109116118125126133134 \\
134135135136\end{array}$ & $\begin{array}{l}909293639535670738083 \\
110111111112\end{array}$ \\
\hline
\end{tabular}

In order to determine the closure of an orbit $\mathcal{O}_{1}^{k}$ we shall employ the following recursive procedure. The centralizer $Z=Z_{K}\left(H^{k}\right)$ is a connected reductive subgroup of $K$ which can be easily determined from the integers $\beta_{i}\left(H^{k}\right)$ given in Table 2 . Furthermore $Z$ is a Levi factor of the parabolic subgroup $Q_{H^{k}}$ of $K$. The centralizer of $E^{k}$ in $Z$ is reductive, and consequently the $\operatorname{PV}\left(Z, \mathfrak{g}_{H^{k}}(1,2)\right)$ is regular [15]. Hence the singular set $S$ of this $\mathrm{PV}$ is a union of irreducible conical hypersurfaces $S_{i}$ defined by equations $f_{i}=0$, where the $f_{i}$ 's are the basic relative invariants of this PV. One knows how to compute the number $m$ of the basic relative invariants [16, Proposition 4]. In particular, $m$ cannot exceed the length of $\mathfrak{g}_{H^{k}}(1,2)$ as a $Z$-module. In all cases that we encounter below $m$ is actually equal to the length of this module. The pair $\left(Q_{H^{k}}, \mathfrak{p}_{2}\left(H^{k}\right)\right)$ is also a PV and its singular set is the union of the hypersurfaces $\hat{S}_{i}=S_{i}+\mathfrak{p}_{3}\left(H^{k}\right)$. In most cases each of the hypersurfaces $\hat{S}_{i}$ contains a dense open $Q_{H^{k}}$-orbit and we are

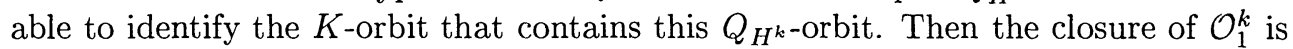
the union of $\mathcal{O}_{1}^{k}$ and the closures of $K$-orbits (of smaller dimension) which contain a dense open subset of one of the hypersurfaces $\hat{S}_{i}$.

We start with the pair $(14,4)$. The PV $\left(Z_{K}\left(H^{14}\right), \mathfrak{g}_{H^{14}}(1,2)\right)$ is irreducible and its singular set $S$ is an irreducible hypersurface. The singular set of $\left(Q_{H^{14}}, \mathfrak{p}_{2}\left(H^{14}\right)\right)$ is the irreducible hypersurface $\hat{S}=S+\mathfrak{p}_{3}\left(H^{14}\right)$. The element

$$
E=\left(X_{114}+X_{-47}\right)+\left(X_{113}\right)+\left(X_{-50}\right)
$$

belongs to $\mathfrak{p}_{2}\left(H^{14}\right) \cap \mathcal{O}_{1}^{11}$ (see Table 4 ). A computation shows that $Q_{H^{14}} \cdot E$ has dimension 35 . As $\mathfrak{p}_{2}\left(H^{14}\right)$ has dimension 36 , this orbit is dense in $\hat{S}$. Consequently

$$
\overline{\mathcal{O}_{1}^{14}}=\mathcal{O}_{1}^{14} \cup \overline{\mathcal{O}_{1}^{11}}
$$

By (4.2) we know that $\mathcal{O}_{1}^{11} \ngtr \mathcal{O}_{1}^{4}$, and so $\mathcal{O}_{1}^{14} \ngtr \mathcal{O}_{1}^{4}$.

Next we consider the pair $(24,19)$. The argument is similar to the one used above. The singular set $S$ of $\left(Z_{K}\left(H^{24}\right), \mathfrak{g}_{H^{24}}(1,2)\right)$ is the union of two irreducible hypersurfaces $S_{1}$ and $S_{2}$. The singular set of $\left(Q_{H^{24}}, \mathfrak{p}_{2}\left(H^{24}\right)\right)$ is also the union of two irreducible hypersurface $\hat{S}_{i}=S_{i}+\mathfrak{p}_{3}\left(H^{24}\right)$. Let $E_{1}$ and $E_{2}$ be the elements from Table 4 in the rows with $i=24$ and $j=23,18$. Hence $E_{1}$ belongs to $p_{2}\left(H^{24}\right) \cap \mathcal{O}_{1}^{23}$ and $E_{2}$ to $\mathfrak{p}_{2}\left(H^{24}\right) \cap \mathcal{O}_{1}^{18}$. A computation shows that the orbits $Q_{H^{14}} \cdot E_{i}$ have dimension 55 . As $\mathfrak{p}_{2}\left(H^{24}\right)$ has dimension 56 , these orbits are dense in $\hat{S}_{i}$. Consequently

$$
\overline{\mathcal{O}_{1}^{24}}=\mathcal{O}_{1}^{24} \cup \overline{\mathcal{O}_{1}^{23}} \cup \overline{\mathcal{O}_{1}^{18}}
$$

By (4.2) we know that $\mathcal{O}_{1}^{23} \ngtr \mathcal{O}_{1}^{19}$, and so $\mathcal{O}_{1}^{24} \ngtr \mathcal{O}_{1}^{19}$.

Let us now consider the pair $(21,11)$. The argument is similar to the one used above but we have to use it several times. We shall indicate only the main steps. 
The singular set of $\left(Q_{H^{21}}, \mathfrak{p}_{2}\left(H^{21}\right)\right)$ is the union of two irreducible hypersurfaces. Let $E_{1}$ be the element from Table 4 in the row with $i=21, j=18$ and let $E_{2}=$ $X_{-8}+X_{-74}+X_{-104}+X_{-118}$. The orbits $Q_{H^{21}} \cdot E_{i}$ are dense in the two hypersurfaces. As $E_{2}$ has type $\left(4 A_{1}\right)^{\prime \prime}$, it belongs (see Table 2 ) to one of the orbits $\mathcal{O}_{1}^{6}, \mathcal{O}_{1}^{7}, \mathcal{O}_{1}^{8}$. We have also computed the dimension of the orbit $G \cdot E_{2}$. Since this dimension is 114 , we obtain another proof (see Table 1 ) that $E_{2}$ belongs to one of the three $K$-orbits mentioned above. (By embedding $E_{2}$ in a suitable normal triple one can show that in fact it belongs to $\mathcal{O}_{1}^{6}$.) As $E_{1} \in \mathcal{O}_{1}^{18}$ and $\overline{\mathcal{O}_{1}^{18}} \supset \mathcal{O}_{1}^{6} \cup \mathcal{O}_{1}^{7} \cup \mathcal{O}_{1}^{8}$, we conclude that

$$
\overline{\mathcal{O}_{1}^{21}}=\mathcal{O}_{1}^{21} \cup \overline{\mathcal{O}_{1}^{18}} \text {. }
$$

The singular set of $\left(Q_{H^{18}}, \mathfrak{p}_{2}\left(H^{18}\right)\right)$ is also the union of two irreducible hypersurfaces. Let $E_{3}$ (resp. $E_{4}$ ) be the element from Table 4 in the row with $i=18$, $j=16$ (resp. $i=18, j=12$ ). Then a computation shows that the orbits $Q_{H^{18}} \cdot E_{3}$ and $Q_{H^{18}} \cdot E_{4}$ have co-dimension 1 in $\mathfrak{p}_{2}\left(H^{18}\right)$, and so they are dense in the two hypersurfaces. Consequently

$$
\overline{\mathcal{O}_{1}^{18}}=\mathcal{O}_{1}^{18} \cup \overline{\mathcal{O}_{1}^{16}} \cup \overline{\mathcal{O}_{1}^{12}}
$$

We now claim that

$$
\overline{\mathcal{O}_{1}^{16}}=\mathcal{O}_{1}^{16} \cup \overline{\mathcal{O}_{1}^{13}}
$$

The argument in this case is more complicated and we shall provide more details. As a $Z_{K}\left(H^{16}\right)$-module, $\mathfrak{g}_{H^{16}}(1,2)$ is the direct sum of two 1-dimensional modules $\left\langle X_{118}\right\rangle$ and $\left\langle X_{-74}\right\rangle$, and the 8-dimensional simple module

$$
\left\langle X_{-98}, X_{-94}, X_{-90}, X_{-87}, X_{-86}, X_{-83}, X_{-78}, X_{-73}\right\rangle .
$$

This PV has 3 basic relative invariants:

$$
f_{1}(X)=a, \quad f_{2}(X)=b, \quad f_{3}(X)=c j-d i+e h-f g,
$$

where

$$
\begin{aligned}
X= & a X_{118}+b X_{-74}+c X_{-98}+d X_{-94}+e X_{-90}+f X_{-87}+g X_{-86} \\
& +h X_{-83}+i X_{-78}+j X_{-73} .
\end{aligned}
$$

If we set $\hat{f}_{i}(Z)=f_{i}(X)$ for $Z=X+Y$ with $X \in \mathfrak{g}_{H^{16}}(1,2)$ and $Y \in \mathfrak{p}_{3}\left(H^{16}\right)$, then we obtain the basic relative invariants of $\left(Q_{H^{16}}, \mathfrak{p}_{2}\left(H^{16}\right)\right)$. The elements $E_{i}^{\prime}$ defined by

$$
\begin{aligned}
& E_{1}^{\prime}=\left(X_{-74}+X_{119}\right)+\left(X_{-73}\right)+\left(X_{-98}\right), \\
& E_{2}^{\prime}=X_{-86}+X_{118}+X_{-87}, \\
& E_{3}^{\prime}=\left(X_{118}+X_{-98}\right)+\left(X_{-47}\right)+\left(X_{-74}\right),
\end{aligned}
$$

belong to the hypersurfaces $\hat{S}_{i}$ defined by $\hat{f}_{i}(Z)=0$ and their $Q_{H^{16} \text {-orbits are dense }}$ in these hypersurfaces. This follows from the fact that each of these three orbits has co-dimension 1 in $\mathfrak{p}_{2}\left(H^{16}\right)$. From Table 4 we see that $E_{2}^{\prime} \in \mathcal{O}_{1}^{13}$. Both $E_{1}^{\prime}$ and $E_{3}^{\prime}$ have type $A_{2}+2 A_{1}$ and so they belong either to $\mathcal{O}_{1}^{10}$ or $\mathcal{O}_{1}^{11}$. It is important to show that 
they both belong to $\mathcal{O}_{1}^{10}$. This can be shown by embedding, say, $E_{1}^{\prime}$ into a principal normal triple $\left(E_{1}^{\prime}, H, F_{1}^{\prime}\right)$ of $A_{2}+2 A_{1}$. An example of such a computation was given in the beginning of this proof. We omit most of the details but we point out that $H \in \mathfrak{h}$ has the following labels:

$$
\alpha_{m}(H)=2,0,0,0,0,-1,2,-4 \quad(1 \leq m \leq 8) .
$$

In the case of $E_{3}^{\prime}$, the corresponding labels are

$$
\alpha_{m}(H)=2,1,-2,0,0,2,-1,-3 \quad(1 \leq m \leq 8) .
$$

As $\mathcal{O}_{1}^{13}>\mathcal{O}_{1}^{10}$, our claim is proved.

The singular set of $\left(Q_{H^{13}}, \mathfrak{p}_{2}\left(H^{13}\right)\right)$ is the union of two irreducible hypersurfaces. Let $E_{4}^{\prime}$ be the element from Table 4 in the row with $i=13, j=10$ and let $E_{5}^{\prime}=$ $X_{-68}+X_{-101}+X_{119}+X_{-15}$. A computation shows that the orbits $Q_{H^{13}} \cdot E_{4}^{\prime}$ and $Q_{H^{13}} \cdot E_{5}^{\prime}$ have co-dimension 1 in $\mathfrak{p}_{2}\left(H^{13}\right)$, and so are dense in these two hypersurfaces. The element $E_{4}^{\prime}$ belongs to $\mathcal{O}_{1}^{10}$ (see Table 4). The element $E_{5}^{\prime}$ has type $\left(4 A_{1}\right)^{\prime \prime}$ and is contained in $\mathfrak{g}_{H^{7}}(1,2)$. Consequently it belongs to $\mathcal{O}_{1}^{7}$. Hence

$$
\overline{\mathcal{O}_{1}^{13}}=\mathcal{O}_{1}^{13} \cup \overline{\mathcal{O}_{1}^{10}} .
$$

Since $\mathcal{O}_{1}^{12} \ngtr \mathcal{O}_{1}^{11}$ by (4.2), it follows from (4.3-6) that $\mathcal{O}_{1}^{21} \ngtr \mathcal{O}_{1}^{11}$.

We move now to the pair $(33,28)$. The singular set of $\left(Q_{H^{33}}, \mathfrak{p}_{2}\left(H^{33}\right)\right)$ is the union of three irreducible hypersurfaces. Let $E_{1}$ and $E_{2}$ be the elements from Table 4 in the rows with $i=33$ and $j=21$ and $j=31$, respectively. Let

$$
E_{3}=\left(X_{-78}+X_{118}+X_{-94}\right)+\left(X_{-79}+X_{104}+X_{-102}\right) .
$$

A computation shows that each of the orbits $Q_{H^{21}} \cdot E_{i}$ has co-dimension 1 in $\mathfrak{p}_{2}\left(H^{33}\right)$ and so is dense in one of these hypersurfaces. Clearly; $E_{1} \in \mathcal{O}_{1}^{21}$ and $E_{2} \in \mathcal{O}_{1}^{31}$. Since $E_{3}$ has type $2 A_{3}$ and belongs to $\mathfrak{g}_{H^{24}}(1,2)$, it follows that it belongs to $\mathcal{O}_{1}^{24}$. Hence

$$
\overline{\mathcal{O}_{1}^{33}}=\mathcal{O}_{1}^{33} \cup \overline{\mathcal{O}_{1}^{21}} \cup \overline{\mathcal{O}_{1}^{31}} \text {. }
$$

The singular set of $\left(Q_{H^{31}}, \mathfrak{p}_{2}\left(H^{31}\right)\right)$ is the union of two irreducible hypersurfaces. Let $E_{4}$ (resp. $E_{5}$ ) be the element from Table 4 in the row with $i=31, j=27$ (resp. $i=31, j=29$ ). Then a computation shows that the orbits $Q_{H^{18}} \cdot E_{4}$ and $Q_{H^{18}} \cdot E_{5}$ have co-dimension 1 in $\mathfrak{p}_{2}\left(H^{31}\right)$, and so they are dense in these two hypersurfaces. Consequently

$$
\overline{\mathcal{O}_{1}^{31}}=\mathcal{O}_{1}^{31} \cup \overline{\mathcal{O}_{1}^{2 \top}} \cup \overline{\mathcal{O}_{1}^{29}}
$$

The $Z_{K}\left(H^{29}\right)$-module $\mathfrak{g}_{H^{29}}(1,2)$ is a direct sum of the 1-dimensional module $\left\langle X_{-74}\right\rangle$ and two nonisomorphic simple 8-dimensional modules:

$$
\begin{aligned}
V_{8} & =\left\langle X_{-73}, X_{-78}, X_{-83}, X_{-86}, X_{-87}, X_{-90}, X_{-94}, X_{-98}\right\rangle, \\
W_{8} & =\left\langle X_{112}, X_{110}, X_{108}, X_{106}, X_{105}, X_{103}, X_{100}, X_{96}\right\rangle .
\end{aligned}
$$


The singular set of $\left(Q_{H^{29}}, \mathfrak{p}_{2}\left(H^{29}\right)\right)$ is the union of three irreducible hypersurfaces $\hat{S}_{i}$ $(i=1,2,3)$. Let $E_{1}^{\prime}$ be the element from Table 4 in the row with $i=29$ and $j=10$. Further, let

$$
\begin{aligned}
& E_{2}^{\prime}=\left(X_{-73}+X_{96}+X_{-74}+X_{113}\right)+\left(X_{-98}\right) \\
& E_{3}^{\prime}=X_{-73}+X_{96}+X_{112}+X_{113}+X_{-11}+X_{-98}
\end{aligned}
$$

A computation shows that the orbits $Q_{H^{29}} \cdot E_{i}^{\prime}$ have co-dimension 1 in $\mathfrak{p}_{2}\left(H^{29}\right)$, and so are dense in the three hypersurfaces. Note that the elements $E_{1}^{\prime}$ and $E_{2}^{\prime}$ are both of type $A_{4}+A_{1}$ and so belong to $\mathcal{O}_{1}^{26}$ (see Table 1). However the closures of their

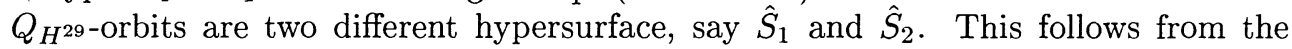
observation that the $V_{8}$-component of $E_{1}^{\prime}$, namely $X_{-98}$, is not generic in the module $V_{8}$ while that of $E_{2}^{\prime}$, namely $X_{-73}+X_{-98}$, is. (The situation is just the opposite for the $W_{8}$-components.) It is not obvious to which $K$-orbit the element $E_{3}^{\prime}$ belongs. But a computation shows that the orbit $G \cdot E_{3}^{\prime}$ has dimension 166 , and so (see Table 1) $E_{3}^{\prime}$ belongs to one of the orbits $\mathcal{O}_{1}^{18}, \mathcal{O}_{1}^{19}, \mathcal{O}_{1}^{20}$. Consequently, the closure of the orbit $Q_{H^{29}} \cdot E_{3}^{\prime}$ is the third hypersurface $\hat{S}_{3}$. As the orbits $\mathcal{O}_{1}^{i}$ for $i=18,19,20$, and 26 have dimensions less then that of $\mathcal{O}_{1}^{28}$, we conclude that $\mathcal{O}_{1}^{29} \ngtr \mathcal{O}_{1}^{28}$. Now (4.7) and (4.8) imply that $\mathcal{O}_{1}^{33} \ngtr \mathcal{O}_{1}^{28}$.

In connection with this case, we make an interesting observation. Consider the $G$-orbits $\mathcal{O}^{28}$ and $\mathcal{O}^{27}$ with Bala-Carter labels $A_{5}$ and $A_{4}+A_{2}$, respectively. By Table 1 we have

$$
\mathfrak{p} \cap \mathcal{O}^{28}=\mathcal{O}_{1}^{29}, \quad \mathfrak{p} \cap \mathcal{O}^{27}=\mathcal{O}_{1}^{28}
$$

Our observation is that

$$
\mathcal{O}_{1}^{29} \ngtr \mathcal{O}_{1}^{28}
$$

although (see Figure 2)

$$
\mathcal{O}^{28}>\mathcal{O}^{27}
$$

The same phenomenon was observed in our previous paper [11].

We next consider the pair $(34,6)$ from the list (4.1). The proof in this case is quite different from the ones above. (A similar proof was used in our paper [10].) The idea is to construct a closed subset of $\mathfrak{p}$ which contains $\mathcal{O}_{1}^{34}$ but not $\mathcal{O}_{1}^{6}$. We begin by observing that, as a $K$-module, $\mathfrak{p}$ is isomorphic to $V \otimes W$ where $V$ is the 56-dimensional simple module for $\mathrm{E}_{7}$ and $W$ is the 2-dimensional simple module for $\mathrm{SL}_{2}$. Furthermore, $\mathfrak{p}=\mathfrak{p}^{+} \oplus \mathfrak{p}^{-}$where $\mathfrak{p}^{+}$resp. $\mathfrak{p}^{-}$is the subspace spanned by the positive resp. negative root spaces in $\mathfrak{p}$. Both $\mathfrak{p}^{+}$and $\mathfrak{p}^{-}$are isomorphic to $V$ as $\mathrm{E}_{7}$-modules. We shall identify $V$ with $\mathfrak{p}^{+}$. An explicit $\mathrm{E}_{7}$-isomorphism $\varphi: \mathfrak{p}^{+} \rightarrow \mathfrak{p}^{-}$ is given by $\varphi(X)=\left[X_{-120}, X\right]$.

Let $\pi: \mathfrak{p} \rightarrow \mathfrak{p}^{+}$be the projector with kernel $\mathfrak{p}^{-}$. Note that $\pi$ commutes with the action of $\mathrm{E}_{7}$. Any $Z \in \mathfrak{p}$ can be written uniquely as $Z=X+\varphi(Y)$ with $X, Y \in \mathfrak{p}^{+}$. The orbit $\mathrm{SL}_{2} \cdot Z$ consists of all vectors

$$
(a X+b Y)+\varphi(c X+d Y)
$$


with $a d-b c=1$. Hence

$$
\pi\left(\mathrm{SL}_{2} \cdot Z\right)=\langle X, Y\rangle \backslash\{0\}
$$

The pair $\left(\mathrm{E}_{7} \times \mathrm{GL}_{1}, \mathfrak{p}^{+}\right)$, where $\mathrm{GL}_{1}$ is the maximal torus of the $\mathrm{SL}_{2}$ factor of $K$ leaving $\mathfrak{p}^{+}$and $\mathfrak{p}^{-}$invariant, is a regular PV (see [15, p. 147]). This PV was studied by S.J. Haris in [12]. The singular set is the hypersurface $S=\mathfrak{p}^{+} \backslash \mathcal{O}_{1}^{6}$ and consists of 4 orbits. Let $E \in \mathcal{O}_{1}^{34}$ be the representative from Table 2 and write $E=X+\varphi(Y)$ where

$$
X=X_{102}+X_{94}+X_{96}, \quad \varphi(Y)=X_{-77}+X_{-68}+X_{-79} .
$$

We have

$$
\pi\left(\mathrm{SL}_{2} \cdot E\right) \subset\langle X, Y\rangle \subset\left\langle X_{102}, X_{94}, X_{96}, X_{100}, X_{93}, X_{95}\right\rangle \subset S .
$$

The last inclusion follows from the fact that the $\left(\mathrm{E}_{7} \times \mathrm{GL}_{1}\right)$-orbit of the element

$$
X_{102}+X_{94}+X_{96}+X_{100}+X_{93}+X_{95}
$$

has dimension 55 and the observation that this orbit contains all linear combinations of the root vectors $X_{102}, X_{94}, X_{96}, X_{100}, X_{93}, X_{95}$ with all coefficients nonzero.

Hence $\pi\left(\mathcal{O}_{1}^{34}\right) \subset S$ and so $\mathcal{O}_{1}^{34} \subset S+\mathfrak{p}^{-}$. Thus $\overline{\mathcal{O}_{1}^{34}} \subset S+\mathfrak{p}^{-}$and consequently $\mathcal{O}_{1}^{6} \not \subset \overline{\mathcal{O}_{1}^{34}}$, i.e., $\mathcal{O}_{1}^{34} \ngtr \mathcal{O}_{1}^{6}$.

It remains to consider the pair $(35,21)$ from the list $(4.2)$. The singular set of $\left(Q_{H^{35}}, \mathfrak{p}_{2}\left(H^{35}\right)\right)$ is the union of three irreducible hypersurfaces. Let $E_{1}$ and $E_{2}$ be the elements from Table 4 in the rows with $i=35$ and $j=34$ and $j=32$, respectively. Let

$$
E_{3}=\left(X_{-84}+X_{96}+X_{-73}+X_{-98}\right)+\left(X_{-99}\right) .
$$

A computation shows that each of the orbits $Q_{H^{35}} \cdot E_{i}$ has co-dimension 1 in $\mathfrak{p}_{2}\left(H^{35}\right)$ and so is dense in one of the hypersurfaces. Clearly, $E_{1} \in \mathcal{O}_{1}^{34}$ and $E_{2} \in \mathcal{O}_{1}^{32}$. Since $E_{3}$ has type $\left(A_{5}+A_{1}\right)^{\prime \prime}$, it follows that it belongs to either $\mathcal{O}_{1}^{30}$ or $\mathcal{O}_{1}^{31}$. (One can show that it belongs to $\mathcal{O}_{1}^{31}$, but we do not need this fact.) Hence

$$
\overline{\mathcal{O}_{1}^{35}}=\mathcal{O}_{1}^{35} \cup \overline{\mathcal{O}_{1}^{34}} \cup \overline{\mathcal{O}_{1}^{32}}
$$

Since $\mathcal{O}_{1}^{34} \ngtr \mathcal{O}_{1}^{21}$ by (4.2), it remains to show that $\mathcal{O}_{1}^{32} \ngtr \mathcal{O}_{1}^{21}$. The $Z_{K}\left(H^{32}\right)$ module $\mathfrak{g}_{H^{32}}(1,2)$ is a direct sum of three 1-dimensional modules $\left\langle X_{104}\right\rangle,\left\langle X_{-74}\right\rangle$, $\left\langle X_{-101}\right\rangle$, and the 8-dimensional simple module

$$
V_{8}=\left\langle X_{96}, X_{100}, X_{103}, X_{105}, X_{106}, X_{108}, X_{110}, X_{112}\right\rangle \text {. }
$$

We shall write an arbitrary element $Z \in \mathfrak{p}_{2}\left(H^{32}\right)$ as $Z=X+Y$ where $X \in \mathfrak{g}_{H^{32}}(1,2)$, $Y \in \mathfrak{p}_{3}\left(H^{32}\right)$, and

$$
\begin{aligned}
X= & a X_{96}+b X_{100}+c X_{103}+d X_{105}+d^{\prime} X_{106}+c^{\prime} X_{108} \\
& +b^{\prime} X_{110}+a^{\prime} X_{112}+u X_{104}+v X_{-74}+w X_{-101} .
\end{aligned}
$$


The basic relative invariants of $\left(Q_{H^{32}}, \mathfrak{p}_{2}\left(H^{32}\right)\right)$ are given by:

$$
\begin{aligned}
& \hat{f}_{1}(Z)=u, \quad \hat{f}_{2}(Z)=v, \quad \hat{f}_{3}(Z)=w \\
& \hat{f}_{4}(Z)=a a^{\prime}-b b^{\prime}+c c^{\prime}-d d^{\prime}
\end{aligned}
$$

The singular set $\hat{S}$ of this PV is the union of the three hyperplanes $\hat{S}_{i}: \hat{f}_{i}(Z)=0$, $i=1,2,3$, and the irreducible quadric $\hat{S}_{4}: \hat{f}_{4}(Z)=0$. Let $E_{1}, E_{3}$, and $E_{4}$ be the elements from Table 4 listed in the rows with $i=32$ and $j=31,30$, and 27, respectively. It is easy to check that $E_{i} \in \hat{S}_{j}$ is true if and only if $j=i$. A computation

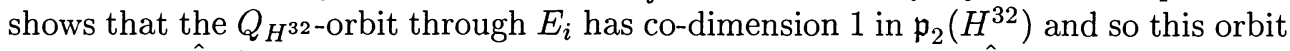
is dense in $\hat{S}_{i}(i=1,3,4)$. The situation with the hyperplane $\hat{S}_{2}$ is quite different. The group $Q_{H^{32}}$ has no open dense orbit in this hyperplane. A computation shows

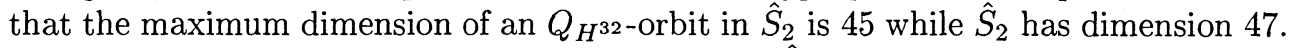
The maximum dimension of an orbit $K \cdot Z$ for $Z \in \hat{S}_{2}$ is 83 and so all these orbits are contained in the closure of $\mathcal{O}_{1}^{31}$ (see Figure 3). Consequently,

$$
\overline{\mathcal{O}_{1}^{32}}=\mathcal{O}_{1}^{32} \cup \overline{\mathcal{O}_{1}^{30}} \cup \overline{\mathcal{O}_{1}^{31}}
$$

Now (4.2) implies that $\mathcal{O}_{1}^{32} \ngtr \mathcal{O}_{1}^{21}$.

5. Appendix. The simple roots $\alpha_{i}, 1 \leq i \leq 8$, are numbered as in Figure 4 .

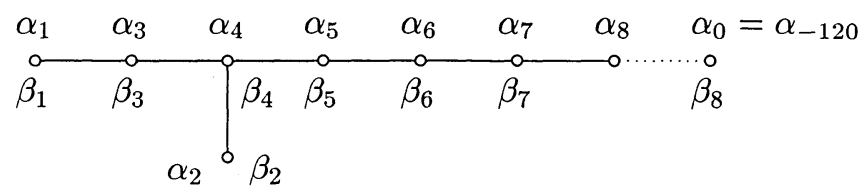

Figure 4: The extended base of $E_{8}$ and a base of $\mathfrak{k}$

In Table 6 we give our enumeration of the positive roots of $E_{8}$. This enumeration is the same as in our paper [8].

Table 6: Positive roots of $\mathrm{E}_{8}$

\begin{tabular}{|cc|cc|cc|cc|}
\hline$i$ & $\alpha_{i}$ & $i$ & $\alpha_{i}$ & $i$ & $\alpha_{i}$ & $i$ & $\alpha_{i}$ \\
\hline 1 & 10000000 & 31 & 10111100 & 61 & 01122210 & 91 & 12232221 \\
2 & 01000000 & 32 & 01121000 & 62 & 01122111 & 92 & 11233221 \\
3 & 00100000 & 33 & 01111100 & 63 & 11232100 & 93 & 12343210 \\
4 & 00010000 & 34 & 01011110 & 64 & 11222110 & 94 & 12243211 \\
5 & 00001000 & 35 & 00111110 & 65 & 11221111 & 95 & 12233221 \\
6 & 00000100 & 36 & 00011111 & 66 & 11122210 & 96 & 11233321 \\
7 & 00000010 & 37 & 11121000 & 67 & 11122111 & 97 & 22343210 \\
8 & 00000001 & 38 & 11111100 & 68 & 01122211 & 98 & 12343211 \\
9 & 10100000 & 39 & 10111110 & 69 & 12232100 & 99 & 12243221 \\
\hline
\end{tabular}


Table 6: (continued)

\begin{tabular}{|cc|cc|cc|cc|}
\hline$i$ & $\alpha_{i}$ & $i$ & $\alpha_{i}$ & $i$ & $\alpha_{i}$ & $i$ & $\alpha_{i}$ \\
\hline 10 & 01010000 & 40 & 01121100 & 70 & 11232110 & 100 & 12233321 \\
11 & 00110000 & 41 & 01111110 & 71 & 11222210 & 101 & 22343211 \\
12 & 00011000 & 42 & 01011111 & 72 & 11222111 & 102 & 12343221 \\
13 & 00001100 & 43 & 00111111 & 73 & 11122211 & 103 & 12243321 \\
14 & 00000110 & 44 & 11221000 & 74 & 01122221 & 104 & 22343221 \\
15 & 00000011 & 45 & 11121100 & 75 & 12232110 & 105 & 12343321 \\
16 & 10110000 & 46 & 11111110 & 76 & 11232210 & 106 & 12244321 \\
17 & 01110000 & 47 & 10111111 & 77 & 11232111 & 107 & 22343321 \\
18 & 01011000 & 48 & 01122100 & 78 & 11222211 & 108 & 12344321 \\
19 & 00111000 & 49 & 01121110 & 79 & 11122221 & 109 & 22344321 \\
20 & 00011100 & 50 & 01111111 & 80 & 12232210 & 110 & 12354321 \\
21 & 00001110 & 51 & 11221100 & 81 & 12232111 & 111 & 22354321 \\
22 & 00000111 & 52 & 11122100 & 82 & 11233210 & 112 & 13354321 \\
23 & 11110000 & 53 & 11121110 & 83 & 11232211 & 113 & 23354321 \\
24 & 10111000 & 54 & 11111111 & 84 & 11222221 & 114 & 22454321 \\
25 & 01111000 & 55 & 01122110 & 85 & 12233210 & 115 & 23454321 \\
26 & 01011100 & 56 & 01121111 & 86 & 12232211 & 116 & 23464321 \\
27 & 00111100 & 57 & 11222100 & 87 & 11233211 & 117 & 23465321 \\
28 & 00011110 & 58 & 11221110 & 88 & 11232221 & 118 & 23465421 \\
29 & 00001111 & 59 & 11122110 & 89 & 12243210 & 119 & 23465431 \\
30 & 11111000 & 60 & 11121111 & 90 & 12233211 & 120 & 23465432 \\
\hline
\end{tabular}

\section{REFERENCES}

[1] D. Barbasch AND M. R. SePANSKI, Closure ordering and the Kostant-Sekiguchi correspondence, Proc. Amer. Math. Soc., 126 (1998), pp. 311-317.

[2] W. M. Beynon AND N. Spaltenstein, Green functions of finite Chevalley groups of type $E_{n}(n=6,7,8)$, J. Algebra, 88 (1984), pp. 584-614.

[3] N. Bourbaki, Groupes et algèbres de Lie, Chap. IV, V, et VI, Hermann, Paris, 1968.

[4] R. W. Carter, Finite Groups of Lie Type: Conjugacy Classes and Complex Characters, J. Wiley, New York, 1985.

[5] B. W. Char, K. O. Geddes, G. H. Gonnet, B. L. Leong, M. B. Monagan, and S. M. Watt, Maple $V$ Language reference Manual, Springer-Verlag, New York, 1991, xv +267 pp.

[6] D. H. Collingwood and W. M. McGovern, Nilpotent Orbits in Semisimple Lie Algebras, Van Nostrand Reinhold, New York, 1993.

[7] D.Ž. ĐoKović, Classification of nilpotent elements in simple exceptional real Lie algebras of inner type and description of their centralizers, J. Algebra, 112 (1988), pp. 503-524.

[8] —, Explicit Cayley triples in real forms of $E_{8}$, Pacific J. Math. 194 (2000), pp. 57-82.

[9] - The closure diagrams for nilpotent orbits of real forms of $F_{4}$ and $G_{2}$, J. Lie Theory, 10 (2000), pp. 491-510.

[10] - The closure diagrams for nilpotent orbits of real forms of $E_{6}$, Preprint, 2000, $33 \mathrm{pp}$.

[11] - The closure diagrams for nilpotent orbits of the real forms $E V I$ and $E$ VII of $E_{7}$, Representation Theory, 5 (2001), pp. 17-42.

[12] S. J. HARIS, Some irreducible representations of exceptional algebraic groups, Amer. J. Math., 93 (1971), pp. 75-106.

[13] H. Matsumoto, Quelques remarques sur les groupes de Lie algébriques réels, J. Math. Soc. Japan, 16 (1964), pp. 419-446.

[14] K. Mizuno, The conjugate classes of unipotent elements of the Chevalley groups $E_{7}$ and $E_{8}$, Tokyo J. Math., 3 (1980), pp. 391-461. 
[15] M. SATo AND T. KimURA, A classification of irreducible prehomogeneous vector spaces and their relative invariants, Nagoya Math. J., 65 (1977), pp. 1-155.

[16] M. SATo, T. Shintani, And M. Muro, Theory of prehomogeneous vector spaces (algebraic part), Nagoya Math. J., 120 (1990), pp. 1-34 (The English translation of Sato's lecture from Shintani's note).

[17] N. Spaltenstein, Classes Unipotents et Sous-groupes de Borel, Lecture Notes in Math. 946, Springer-Verlag, Berlin-Heidelberg-New York, 1982.

[18] M. A. A. VAN LeEUWEN, A. M. Cohen, AND B. Lisser, "LiE", a software package for Lie group theoretic computations, Computer Algebra Group of CWI, Amsterdam, The Netherlands, 1996. 